\title{
Contributions to the Relativistic Mechanics of Continuous Media
}

\author{
Jürgen Ehlers ${ }^{1}$
}

Received July 12, 1993

\begin{abstract}
This is a translation from German of an article originally published in Proceedings of the Mathematical-Natural Science Section of the Mainz Academy of Science and Literature, Nr. 11, 1961 (pp. 792-837) (printed by Franz Steiner and Co, Wiesbaden), which is Paper IV in the series "Exact Solutions of the Field Equations of General Relativity Theory" by Pascual Jordan, Jürgen Ehlers, Wolfgang Kundt and Rainer K. Sachs. The translation has been carried out by G. F. R. Ellis (Department of Applied Mathematics, University of Cape Town), assisted by P. K. S. Dunsby, so that this outstanding review paper can be readily accessible to workers in the field today. As far as possible, the translation ${ }^{2}$ has preserved both the spirit and the form of the original paper. ${ }^{3}$ Despite its age, it remains one of the best reviews available in this area.
\end{abstract}

\section{INTRODUCTION}

The description of matter in the General Theory of Relativity can be carried out with the help either of a model of point masses, or a model of a continuously spread out medium. Given the present state of knowledge,

1 Present address: Max Planck Institute for Astrophysics, 85740 Garching-beiMünchen, Germany

2. We thank Dr. Ehlers for checking and correcting the English version.

3 In accord with present day usage, the covariant derivative is represented by a semicolon and partial derivative by a comma, instead of the double vertical line and single vertical line used for these quantities in the original paper. Thus $T_{a b ; c} \equiv T_{a b|| c}$, $T_{a b, c} \equiv T_{a b \mid c}$. 
only the second method of description can be carried out in a objectionfree mathematical way, since no one has so far succeeded even in defining the concept of a "singularity of a metric field that describes a particle", much less in deducing the consequences of such a definition.

From the physical viewpoint there is no decisive objection that gives fully convincing reasons to consider the first model as the more suitable. Indeed in our opinion this is not the case; for the considerations that led Einstein to setting up his theory related to macroscopic processes, and whether the theory can contribute something to the understanding of microphysical elementary processes is at the present time undecided. (Pauli remarked in the introduction to the new edition of his Relativity Theory appearing in 1958, "These differences of opinion are merging into the great open problem of the relation of relativity theory to quantum theory, which will presumably occupy physicists for a long time to come. In particular a clear connection between the general theory of relativity and quantum mechanics is not yet in sight.") In a macroscopic theory however there can be no doubt that the hydrodynamic (or elasto-mechanical) description should be regarded as primary and the point-mechanical as an approximating simplification. Hydrodynamics must therefore be considered as an essential part of gravitational theory.

While the formal carrying over of the special-relativistic expressions for the energy tensor corresponding to hydrodynamics and the conservation equations (replacing the ordinary by covariant divergence) was already carried out by Einstein in 1916, the attempt to develop a systematic general relativistic hydrodynamics was first undertaken in a beautiful work by J. L. Synge in 1937, and these investigations were carried forward with other methods by A. Lichnerowicz; the results of the last named author are presented in his volume on relativity theory that appeared in 1955 . The works of these authors stimulated the present investigations and laid their essential foundations. We will not refer to the contributions of other authors here, but rather at the appropriate place in this work.

The aim of this work is to communicate a series of new results in the framework of a comprehensive presentation that combines in a unified viewpoint the contributions of various authors. This seems all the more appropriate because the standard presentations of relativity theory-with the exception of the above-named book of Lichnerowicz-at best skim over this important and beautiful part of Einstein's theory. ${ }^{4}$ Furthermore

\footnotetext{
4 Some important remarks are contained in the recent book by J. L. Synge [35], whose geometric presentation of relativity theory serves as a model for the present work, and in the book of V. A. Fock [29]. However these remarks do not add up to a
} 
no presentation exists that deals with the kinematic, mechanical, thermodynamic, and kinetic gas theory aspects, linked together and with reference to special solutions of the field equations, as is done here, naturally with restriction to suitably selected topics. (The treatment of specific solutions in particular is only sketched for the case of incoherent matter, but the foundations are developed for a corresponding description of solutions with ideal fluids that will follow.)

In Section 2 neither (dynamic) conservation theorems nor field equations are supposed; rather the general geometric properties of a congruence of timelike curves in a normal-hyperbolic Riemannian space are investigated.

After an illustrative derivation of the kinematic basic variables of the theory, which completes the corresponding investigations by Synge and Lichnerowicz, in the second subsection special flows are characterised.

The identities developed in the third subsection are used in the fourth paragraph for derivation of vorticity theorems. Furthermore stationary and static space-times are characterised by the test-particle relative motions that are possible in them, and a new criterion for their Petrov type is proved.

The kinematic characterisation of cosmological models in the fifth subsection shows the usefulness of the hydrodynamic concepts and theorems for the derivation or illustrative interpretation of particular line-elements. The derivation given there of the homogeneous-isotropic models is simpler than that of Robertson and Walker, usually referred to in the literature, despite the weaker presuppositions made here.

Section 3 begins with a new version of the Weyl-Pauli way of motivating the Einstein Field Equations. The two following subsections contain a derivation for the simplest cases of the thermo-hydrodynamic foundations. The general relativistic 'Navier-Stokes' equation (76) and the 'Poisson' equation (82) have not been given up to this point.

The theory of an ideal gas given in the fourth paragraph is different from that recently published by Synge in 1958, in that here the Boltzmann equation is placed at the beginning, then an H-theorem proved and from this the equilibrium distribution won 'dynamically', while Synge (like earlier authors) uses the Boltzmann counting method (with an additional ad hoc assumption). Furthermore the determination of reversible flows in a gravitational field (Theorem 3.4.3), which is of cosmological interest, is new, as is the related gas-kinetic derivation of the Tolman law concerning its relation with the gravitational (and so also centrifugal) potential. Re- 
marks by A. H. Taub [37] and M. Sasaki [38] have stimulated this part of the work.

The last subsection contains general statements about the motion of pressure-free matter and a hydrodynamic description of the known exact solutions. This subsection also contains some new remarks: it seems to us more significant that the geometric presentation, avoiding a purely formal starting point, is significantly simpler than the usual ones and at the same time leads to a better understanding of the matter concerned.

To conclude these introductory remarks I must stress that-as is shown also in this work-a truly physical understanding of Einstein's theory that is so convincing in its foundations is only now gradually emerging. The wish to contribute to this clarification has led to this work.

\section{Conventions}

Equality by definition: $\equiv$. Proportionality: .

Tensor indices: $a, b, c, \ldots=1,2,3,4 ; \lambda, \mu, \nu \ldots=1,2,3$.

Symmetrisation: ( ), e.g. $F_{(a b)} \equiv \frac{1}{2}\left(F_{a b}+F_{b a}\right)$.

Antisymmetrisation: [ ], e.g. $F_{[a b]} \equiv \frac{1}{2}\left(F_{a b}-F_{b a}\right)$.

Metric tensor: $g_{a b}$, Signature +++- .

Riemann tensor: $R_{a b c d}$.

Ricci tensor: $R_{a b} \equiv R_{a c b}^{c}$, trace: $R \equiv R_{a}^{a}$.

Einstein tensor: $G_{a b} \equiv R_{a b}-\frac{1}{2} R g_{a b}, G \equiv G^{a}{ }_{a}$.

Weyl tensor( = Conformal tensor): $C_{a b c d}$.

Partial differentiation w.r.t. $x^{c}$ : for example $F_{a b, c}$.

Covariant differentiation w.r.t. $x^{c}$ : for example $F_{a b ; c} ; F_{a b ; c d}=F_{a b ; c ; d}$.

Covariant differentiation along $x^{a}(\lambda): \stackrel{\lambda}{\nabla} k_{a}=k_{a ; b}\left(d x^{b} / d \lambda\right)$.

Conventions regarding units: Speed of light $c \equiv 1$, Newtonian Gravitational constant $\gamma \equiv 1 / 8 \pi$, thus Einstein gravitational constant $\kappa \equiv 1$.

\section{KINEMATICS}

\subsection{Fundamental concepts. Decomposition of the velocity gra-} dient

The history of a continuously spread out body is described in relativity theory by a three-dimensional family of timelike curves, the worldlines of the matter elements. With respect to an arbitrary local coordinate system,

$$
x^{a}=x^{a}\left(y^{\alpha}, s\right)
$$

is a parametric representation of the family: the $y^{\alpha}$ designate the matter 
elements, $s$ the proper time along their worldlines. ${ }^{5}$ Then

$$
u^{a} \equiv \frac{\partial x^{a}}{\partial s} \equiv \dot{x}^{a} \quad\left(u^{a} u_{a}=-1\right)
$$

is the normalised tangent vector to the curve, usually referred to as the four-velocity of the substance element.

If

$$
\delta \equiv \delta y^{\nu} \frac{\partial}{\partial y^{\nu}}
$$

means the variation across the world lines and () the covariant derivative with respect to $s$ along the world lines, then

$$
\left(\delta x^{a}\right)^{\cdot}=u_{; b}^{a} \delta x^{b}
$$

for both sides are vectors, and when we use locally flat coordinates (4) expresses the equality of certain second partial derivatives of the functions (1).

The tensor

$$
h_{b}^{a} \equiv \delta_{b}^{a}+u^{a} u_{b}
$$

projects the tangent vector-space at each point perpendicularly onto the three-dimensional subspace orthogonal to $u^{a}$. The vector

$$
\delta_{\perp} x^{a}=h_{b}^{a} \delta x^{b}
$$

is the position vector of the particle $\left(y^{\alpha}+\delta y^{\alpha}\right)$ with respect to the particle $\left(y^{\alpha}\right)$, and its Fermi derivative

$$
v^{a} \equiv h_{b}^{a}\left(\delta_{\perp} x^{b}\right)^{\prime}
$$

is (by definition) the velocity of the particle $\left(y^{\alpha}+\delta y^{\alpha}\right)$ relative to the particle $\left(y^{\alpha}\right)$.

The (absolute) acceleration of the substratum elements is given by the spacelike vector field

$$
\dot{u}^{a} \equiv u_{; b}^{a} u^{b} \quad\left(u_{a} \dot{u}^{a}=0\right)
$$

(In differential geometric terms, $\dot{u}^{a}$ is the first curvature vector of the corresponding curve.)

\footnotetext{
The transformation (1) corresponds to the transformation in classical mechanics from Lagrangian to Eulerian coordinates.
} 
From eqs. (4)-(8) one can derive the velocity field in an infinitesimal neighbourhood of a material element:

$$
v^{a}=u_{; b}^{a} \delta_{\perp} x^{b}
$$

According to the pattern of classical hydrodynamics we decompose the tensor $u^{a}{ }_{; b} h_{c}^{b}$, that brings about the transformation $\delta_{\perp} x^{a} \rightarrow v^{a}$, into irreducible parts (with respect to the rotation group):

$$
u_{a ; c} h_{b}^{c}=\omega_{a b}+\sigma_{a b}+\frac{1}{3} \Theta h_{a b}
$$

where

$$
\omega_{(a b)}=\sigma_{[a b]}=0, \quad \sigma_{a}^{a}=0, \quad \omega_{a b} u^{b}=\sigma_{a b} u^{b}=0 .
$$

The infinitesimal transformation that the vector-space $\left\{\delta_{\perp} x^{b}\right\}$ experiences in the time interval $d s$ decomposes according to the preceding expressions into a rotation $\omega^{a}{ }_{b} \delta_{\perp} x^{b}$, a rotation-free, volume-preserving shearing $\sigma_{b}^{a} \delta_{\perp} x^{b}$, and a rotation-free similarity transformation $\frac{1}{3} \Theta \delta_{\perp} x^{a}$. The distances $\delta l \equiv\left(g_{a b} \delta_{\perp} x^{a} \delta_{\perp} x^{b}\right)^{1 / 2}=\left(h_{a b} \delta x^{a} \delta x^{b}\right)^{1 / 2}$ between neighbouring particles alter during this time according to the equation

$$
\frac{(\delta l)}{\delta l}=\frac{1}{3} \Theta+\sigma_{a b} e^{a} e^{b}, \quad\left(e^{a} \equiv \frac{\delta_{\perp} x^{a}}{\delta l}, e_{a} e^{a}=1\right)
$$

that follows from (7),(9)-(11), and the directions to the neighbouring particles according to

$$
h_{b}^{a} \dot{e}^{b}=\left(\omega^{a}{ }_{b}-\sigma_{b}^{a}-\sigma_{c d} e^{c} e^{d} \delta^{a}{ }_{b}\right) e^{b} .
$$

According to (12) and $\sigma^{a}{ }_{a}=0$, if $\overline{\delta l}$ means a direction average and $\delta V$ the volume of an infinitesimal set of substratum particles,

$$
\Theta=3 \frac{\overline{(\delta l)}}{(\delta l)}=\frac{(\delta V)}{\delta V} .
$$

As (13) shows, $h_{b}^{a} \dot{e}^{b}=\omega^{a}{ }_{b} e^{b}$ holds exactly when $e^{a}$ lies in a principal shearing direction. Therefore that material orthogonal triad of vectors which at any time $s$ coincides with the main shearing axes experiences in the time interval $(s, s+d s)$ the infinitesimal rotation determined by $\omega^{a}{ }_{b}{ }^{6}$

6 Through this, in general the triad rotates itself out of the main shear directions. An exception is given in Corollary 2 to Theorem 2.4.2 in subsection 2.4. 
From the decomposition formulae (10) and the conditions (11) one can derive the following expressions for the vortex velocity $\omega_{a b}$, the shearing velocity $\sigma_{a b}$, and the expansion velocity $\Theta$ :

$$
\begin{gathered}
\omega_{a b}=u_{[a ; b]}-\dot{u}_{[a} u_{b]}, \\
\sigma_{a b}=u_{(a ; b)}-\dot{u}_{(a} u_{b)}-\frac{1}{3} \Theta h_{a b}, \\
\Theta=u_{; \alpha}^{a} .
\end{gathered}
$$

Instead of the vorticity tensor $\omega_{a b}$ one can introduce the vorticity vector

$$
\omega^{a} \equiv \frac{1}{2} \eta^{a b c d} u_{b} \omega_{c d}=\frac{1}{2} \eta^{a b c d} u_{b} u_{c, d} \quad\left(u_{a} \omega^{a}=0\right) ;
$$

$\omega^{a}$ is dual to $\omega_{a b}$ in the rest-space determined by $u^{a}$ :

$$
\omega_{a b}=\eta_{a b c d} \omega^{c} u^{d} .
$$

The consequent equation

$$
\omega_{a b} \omega^{b}=0
$$

implies, according to (9),(10), that $\omega^{a}$ determines the rotation axis. According to (18) the vector $\omega^{a}$ is spacelike, and by (20) lies orthogonal to the plane determined by the simple spacelike bivector $\omega_{a b}$.

A family of timelike curves in general has exactly nine invariants of the first order (independent of each other): the six independent components of the vectors $\dot{u}^{a}$ and $\omega^{a}$ relative to the eigenvector basis of the tensor $\sigma_{b}^{a}$, two independent eigenvalues ${ }^{7}$ of $\sigma^{a}{ }_{b}$, and $\Theta$. These magnitudes determine (for a given metric $g_{a b}$ ) the family of curves in the infinitesimal first order neighbourhood of a point uniquely up to homogeneous Lorentz transformations, as follows from the relation

$$
u_{a ; b}=\omega_{a b}+\sigma_{a b}+\frac{1}{3} \Theta h_{a b}-\dot{u}_{a} u_{b}
$$

which is equivalent to (10).

In addition to $\Theta$ we wish to explicitly introduce for later application the scalars

$$
\dot{u} \equiv\left(\dot{u}_{a} \dot{u}^{a}\right)^{1 / 2}, \omega \equiv\left(\omega_{a} \omega^{a}\right)^{1 / 2}=\left(\frac{1}{2} \omega_{a b} \omega^{a b}\right)^{1 / 2}, \sigma \equiv\left(\frac{1}{2} \sigma_{a b} \sigma^{a b}\right)^{1 / 2} .
$$

These are non-negative and vanish only at the same time as their corresponding tensors.

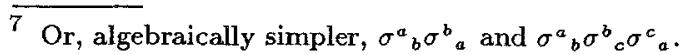


While the physical meaning of the deformation of a substratum element described by the formulae (12),(14) is immediately determined by the axiomatically laid down interpretation of the metric fundamental form $g_{a b} d x^{a} d x^{b}$, the definition of a rotation given above contains an implied convention: this rotation relates to a Fermi-transported triad of vectors that can be thought of as carried along in the rest-frame of the central particle $\left(v^{a}=0\right.$ in (7) means that $\delta_{\perp} x^{a}$ is Fermi-transported). How such a rotation can make itself dynamically felt, can naturally not be decided in a purely kinematical manner.

\subsection{Special Flows. Redshift}

With the help of the concepts introduced in the previous subsection, and with an eye on suitable applications, it is possible to introduce particular designations for some types of flows.

We speak of an inertial flow if the substratum elements follow the "inertial guiding field", i.e. when the matter world lines are geodesic $(\dot{u}=0)$. A vortex-free, or irrotational flow is characterised by $\omega=0$, a volumepreserving, or isochoric one by $\Theta=0$. If infinitesimal substratum elements remain similar to themselves under the action of the flow, for which according to (12) $\sigma=0$ is characteristic, a shear-free flow (isotropic expansion or compression) is taking place. If a flow is isochoric and shear-free, thus when all the distances of neighbouring particles are constant in time, the flow is rigid $(\Theta=\sigma=0)$.

Symmetry properties of a flow determined by a curve family $K$ in the space-time $W$ (or in a region of $W$ ) are described by groups ${ }^{8}$ of isometric (or conformal) mappings of $W$ into itself, which transform $K$ into itself. If $\xi^{a}$ is the generating vector of a one-dimensional Lie Group $G_{1}$ and $u^{a}$ the tangent vector of a family of curves $K$, the invariance of $K$ under $G_{1}$ is expressed by

$$
u_{a ; b} \xi^{b}-u_{b} \xi_{; a}^{b}=0 .
$$

If $K$ is invariant under an isometry group $G_{1}$ and the trajectories of $G_{1}$ in $W$ are timelike, the flow is called stationary, and in the special case of hypersurface-orthogonal trajectories, static. If $K$ is stationary and $\xi^{a} \sim$ $u^{a}$, the flow will be named isometric, and in the more general case when $\xi^{a} \sim u^{a}$ and $G_{1}$ is conformal, it will be called conformal.

The following statements are direct consequences of the definitions.

i. Inertial flows are characterised by the equation

$$
\omega_{a b}=u_{[a ; b]} \text {. }
$$

\footnotetext{
8 Among these groups we also include local Lie groups, which actually are most important for us.
} 
(We display this simple consequence because, while (24) is the formal analogue of the equation $\vec{\omega}=\frac{1}{2}$ rot $\vec{v}$ of classical mechanics, that analogue only holds in restricted cases. According to $(18),\left(\omega^{\nu}\right)=\frac{1}{2}$ rot $\vec{v}$ always holds in the instantaneous rest-system of a substratum element, and this corresponds in content to the usual formula).

ii. A flow is vortex-free exactly when there are (necessarily spacelike) orthogonal hypersurfaces to the matter world-lines [3], i.e. when (locally) there exists a non-constant scalar $t$ such that ${ }^{9}$

$$
\dot{t} u_{a}=-t_{, a} \quad\left(\Leftrightarrow h_{a}^{b} t_{, b}=0\right) .
$$

Equation (25) is at the same time characteristic of the fact that the time functions defined along the matter world lines are synchronous in Einstein's sense. ${ }^{10}$ Such a global time coordinate $t$ agrees with the proper-time $s$ everywhere only in the case of inertial flows; for such vortex-free flows $u_{a}=-t, a$ and $t$ is a solution of the Hamilton-Jacobi equation

$$
t_{, a} t^{, a}+1=0
$$

for free particles in a gravitational field.

iii. Vortex-free flows are volume-preserving precisely when the orthogonal hypersurfaces to the streamlines are minimal [2] $\left(u^{a}{ }_{; a}=0\right.$ is in fact the condition for the vanishing of the first variation of the volume of the hypersurfaces orthogonal to $u^{a}$ ).

A similar statement, useful for the geometry of static matter fields, is the following.

iv. A vortex-free flow is rigid precisely when its orthogonal hypersurfaces are totally geodesic. (Namely let $\omega=0$ and $x^{a}(\lambda)$ a geodesic, $d x^{a} / d \lambda=t^{a}$. Then

$$
\frac{d}{d \lambda}\left(u_{a} t^{a}\right)=u_{a ; b} t^{a} t^{b}=\left(\sigma_{a b}+\frac{1}{3} \Theta h_{a b}-\dot{u}_{a} u_{b}\right) t^{a} t^{b}
$$

so the property $u_{a} t^{a}=0$ propagates itself along exactly when $\sigma=\Theta=0$.)

v. Conformal flows are characterized by

$$
\sigma=0, \quad\left(\dot{u}_{[a}-\frac{1}{3} \Theta u_{[a}\right)_{; b]}=0 .
$$

9 We use the fact that $u_{a} d x^{a}$ has an integrating factor precisely when $u_{[a} u_{b, c]}=0$.

10 This synchronisation condition is usually formulated non-covariantly and without recognition of its world-geometric meaning, see e.g.Ref. 1, pp. 259,260. 
For them there exists a scalar $U$ such that

$$
\dot{u}_{a}-\frac{1}{3} \Theta u_{a}=U_{, a} \quad\left(\dot{U}=\frac{1}{3} \Theta\right) .
$$

(The conditions (27) show that a scalar $U$ exists such that $\xi^{a} \equiv e^{U} u^{a}$ obeys the conformal Killing equation $\xi_{(a ; b)}=\left(e^{U}\right)^{j} g_{a b}$.)

This implies the special case

vi. Isometric flows are characterised by

$$
\sigma=\Theta=0, \quad \dot{u}_{[a ; b]}=0 .
$$

For them there thus exists an "acceleration potential"

$$
\dot{u}_{a}=U_{, a} .
$$

[(29) means that a Killing vector is collinear with $u^{a}$, namely $\xi^{a} \equiv e^{U} u^{a}$ with $U$ according to (30)].

The relative motion of two particles and the "absolute acceleration" of each of them described by $\dot{u}_{a}$ show up e.g. in the wavelength change that occurs when one of the particles emits monochromatic light and the other receives it. We will suppose (in view of application to cosmological models) that the matter elements of the substratum we are considering send out and receive light, and wish to express $d \lambda / \lambda$ for the light that one particle receives from its neighbour, in terms of the kinematic quantities of the flow.

A light ray is described in geometric optics by a geodesic null line ${ }^{11}$ $x^{a}(v): k^{a} \equiv d x^{a} / d v, k_{a} k^{a}=0, \stackrel{k}{\nabla} k^{a}=0$. For monochromatic light $k^{a}$ can and will be chosen so that, for an observer with 4-velocity $u^{a}$, $-k_{a} u^{a}=2 \pi / \lambda$ (see, for example, Ref. 5, p.16). Therefore according to (21), along a light ray in our substratum we have:

$$
\stackrel{k}{\nabla}\left(-u_{a} k^{a}\right)=-u_{a ; b} k^{a} k^{b}=-\left(\sigma_{a b}-\frac{1}{3} \Theta h_{a b}-\dot{u}_{a} u_{b}\right) k^{a} k^{b} .
$$

Furthermore (see Figure 1)

$$
\delta l^{2}=h_{a b} d x^{a} d x^{b}=\left(u_{a} k^{a}\right) d v^{2}=\left(\frac{2 \pi}{\lambda}\right) d v^{2} .
$$

This leads to

$$
\frac{d \lambda}{\lambda}=\frac{-\lambda}{2 \pi} d\left(\frac{2 \pi}{\lambda}\right)=\left(\sigma_{a b} e^{a} e^{b}-\frac{1}{3} \Theta\right) \delta l-\dot{u}_{a} \delta_{\perp} x^{a},
$$

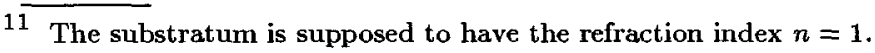


and so from (12) finally

$$
\frac{d \lambda}{\lambda}=(\delta l)-\dot{u}_{a} \delta_{\perp} x^{a}
$$

From this we draw two conclusions. Firstly we conclude through (12):

vii. For a substratum particle receiving light, $d \lambda / \lambda$ is independent of the direction of the infinitesimally neighbouring radiating particles, precisely when its world line $L$ is geodesic and the shear velocity vanishes along $L$; then

$$
\frac{\delta \lambda}{\lambda}=\frac{1}{3} \Theta \delta l
$$

Secondly we combine (27) with remark v., and obtain viii. On a conformal flow $\lambda e^{-U}$ is constant along a lightray. ${ }^{12}$

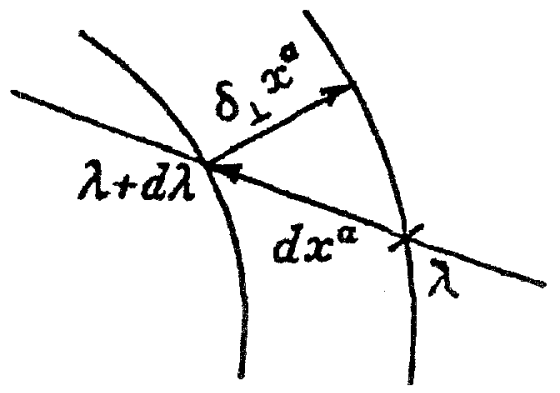

Figure 1.

The last conclusion contains the theory of redshift in stationary and static fields and in the Friedmann cosmological models (see subsection 2.5). In the first case $\Theta=0, \dot{u} \neq 0$ and $U$ (by definition) is the scalar gravitational potential, ${ }^{13}$ and in the last case $\Theta \neq 0, \dot{u}=0$ and $e^{U}$ according to (28) is proportional to the radius of curvature of the space (see subsection 2.5 ), if in both cases the timelike orbits of the isometry (resp. conformal) group are taken as the world-lines of the substrate.

12 This follows directly from the fact that the scalar product of the generating vector of an infinitesimal conformal transformation and the (parallely propagated) tangent vector of a null geodesic is constant along the geodesic.

This definition is validated through (30) and proves itself both suitable and useful. In this connection see (82), Theorem 3.3.2, Theorem 3.4.4, and Ref. 32, Ch.2. 


\subsection{Differential Identities for the Kinematic Quantities}

The derivatives of the kinematic quantities introduced in subsection 2.1 are not independent of each other. Rather, they fulfil certain identities into which inter alia the curvature tensor of space-time enters. These equations are useful for the derivation of propagation and conservation theorems, and for the investigation of the relations between field equations and families of curves.

First we determine the relative acceleration of neighbouring particles. We define it analogously to the relative velocity $v^{a}$ [see (7)] through

$$
b^{a} \equiv h_{b}^{a} \dot{v}^{b}
$$

and obtain for it from (9), using the Ricci identity, the expression

$$
b^{a}=\left(R_{b c d}^{a} u^{b} u^{d}+h_{b}^{a} \dot{u}_{; c}^{b}+\dot{u}^{a} \dot{u}_{c}\right) \delta_{\perp} x^{c}
$$

which generalises the well known formula for geodesic deviation [6].

The first term on the right hand side of (34) brings to light the influence of the 'world' curvature (in Einstein's theory: the gravitational field gradient) on the relative acceleration. The tensor $R_{a b c d} u^{d}$ contained in it can be expressed in terms of the first derivatives of the kinematic quantities $\omega_{a b}, \sigma_{a b}, \Theta$

$$
\begin{aligned}
\frac{1}{2} R_{a b c d} u^{d}= & \omega_{c[a ; b]}+\sigma_{c[a ; b]}+\frac{1}{3} h_{c[a} \Theta_{, b]}-\dot{u}_{c ;[b} u_{a]} \\
& +\frac{1}{3} \Theta\left(u_{c} \omega_{a b}-u_{c} \dot{u}_{[a} u_{b]}-u_{[a} \omega_{b] c}\right. \\
& \left.+\sigma_{c[b} u_{a]}+\frac{1}{3} \Theta g_{c[b} u_{a]}\right)-\dot{u}_{c}\left(\omega_{a b}-\dot{u}_{[a} u_{b]}\right)
\end{aligned}
$$

By contraction one obtains from this $R_{a b} u^{b}$ and its components $R_{a b} u^{a} u^{b}, h^{a b} R_{b c} u^{c}$. After simplification by partial differentiation, where the formulae (11),(21),(22) are used, the following formulae arise:

$$
R_{a b} u^{a} u^{b}=\dot{\Theta}+\frac{1}{3} \Theta^{2}-\dot{u}_{; a}^{a}+2\left(\sigma^{2}-\omega^{2}\right)
$$

and

$$
h^{a b} R_{b c} u^{c}=h_{b}^{a}\left(\omega^{b c}{ }_{; c}-\sigma^{b c}{ }_{; c}+\frac{2}{3} \Theta^{, b}\right)+\left(\omega^{a}{ }_{b}+\sigma^{a}{ }_{b}\right) \dot{u}^{b} .
$$

Finally in order to express the tensor $R_{a b c d} u^{b} u^{d}$ that occurs in (35) in terms of the kinematic quantities, we multiply (35) by $u^{b}$ and simplify the still very complicated expression by symmetrising with respect to indices 
$a, c$ and multiplying by $h_{e}^{a} h_{f}^{c}$, which does not alter the left hand side. We obtain

$$
\begin{aligned}
R_{a b c d} u^{b} u^{d}= & \omega_{a} \omega_{c}-\omega^{2} h_{a c}+\sigma_{a b} \sigma^{b}{ }_{c}+\frac{\ddot{l}}{l} h_{a c} \\
& -\dot{u}_{a} \dot{u}_{c}+h_{a b} h_{c d}\left(l^{-2}\left(l^{2} \sigma^{b d}\right)-\dot{u}^{(b ; d)}\right)
\end{aligned}
$$

Here we have also introduced a useful length scale l by

$$
\frac{i}{l} \equiv \frac{1}{3} \Theta \Rightarrow \frac{1}{3}\left(\dot{\Theta}+\frac{1}{3} \Theta^{2}\right)=\frac{\ddot{l}}{l}
$$

( $l$ is only determined up to a scalar factor that is constant along the flow lines. ${ }^{14}$ )

While the equations (36) and (38) contain the derivatives of the quantities $\Theta$ and $\sigma_{a b}$ respectively, thus far $\dot{\omega}^{a}$ does not occur. ${ }^{15}$ One can however derive a propagation equation for the vorticity vector from the identity $R_{a[b c] d} u^{a} u^{d}=0$ by some transformations, or by direct calculation from the definition (18), which is comparatively simple:

$$
h_{b}^{a}\left(l^{2} \omega^{b}\right)=\sigma_{b}^{a} l^{2} \omega^{b}+\frac{l^{2}}{2} \eta^{a b c d} u_{b} \dot{u}_{c ; d}
$$

or equivalent to this

$$
h_{b}^{a}\left(l^{3} \omega^{b}\right)^{\cdot}=u_{; b}^{a} l^{3} \omega^{b}+\frac{l^{3}}{2} \eta^{a b c d} u_{b} \dot{u}_{c ; d}
$$

It implies for the vorticity scalar

$$
l^{-4}\left(l^{4} \omega^{2}\right)^{j}=2 \sigma_{a b} \omega^{a} \omega^{b}+\omega_{a b} \dot{u}^{a ; b} .
$$

For completeness we give also an identity, somewhat surprising in view of classical hydrodynamics, that follows from the definition of the vorticity vector:

$$
\omega^{a} ; a=2 \dot{u}_{a} \omega^{a} .
$$

$1 \longdiv { 4 }$ Flow lines $\equiv$ matter world lines.

15 It is clear that there is no propagation equation for $\dot{u}^{a}$ because $u^{a}, \dot{u}^{a}, \ddot{u}^{a}$ can be given arbitrarily along a curve independently of the neighbouring curves. 


\subsection{Vorticity theorems and theorems for rigid motions}

As a first application of the formulae collected together in the last section, we will prove some theorems that correspond to the vorticity theorems of classical hydrodynamics.

We designate as vortex lines of a flow, the (spacelike) curves that have the vorticity vector as their tangent vector; according to (8) they cut the flow-lines orthogonally.

The first result is the following lemma.

Lemma 2.4. The flow lines are geodesic with respect to the metric $\vec{g}_{a b}=$ $w^{2} g_{a b}(w>0)$ precisely when (with respect to $\left.g_{a b}\right)$

$$
\dot{u}_{a}=-h_{a}^{b}(\log w)_{, b}
$$

holds.

The proof follows for example from the fact that (44) represents the Euler equation for the variational problem

$$
\delta \int w d s=0
$$

According to (25) and (28), eq. (44) will hold in particular for irrotational and for conformal flows.

We recall further that two vector fields $\xi^{a}, \eta^{a}$ span 2-surfaces if and only if their commutator $\xi^{a}{ }_{, b} \eta^{b}-\eta^{a}{ }_{, b} \xi^{b}$ is (pointwise) linearly dependent on them. From this and (41) there follows

Theorem 2.4.1. The flow- and vortex-lines span surfaces ("vortex surfaces") exactly when

$$
\omega^{[b} \eta^{c] d e f} u_{d} \dot{u}_{e ; f}=0
$$

holds. ${ }^{16}$

The property stated in the theorem means that the vortex lines consist of the same substratum particles at all times ("material" conservation of vortex lines.)

Condition (46) is in particular fulfilled for inertial flows, and more generally for flows satisfying (44), since the latter equation implies that

$$
\frac{1}{2} \eta^{a b c d} u_{b} \dot{u}_{c ; d}=-\frac{\dot{w}}{w} \omega^{a}
$$

16 This and the following theorem agree completely with the vorticity theorems given by Synge [3], Lichnerowicz [2], Foures-Bruhat [7], Gödel [8], Schücking and Heckmann [9]. 
This equations permits one to simplify (40),(41) and to conclude

Theorem 2.4.2. In a flow satisfying (44) the (equivalent) propagation equations

$$
h_{b}^{a}\left(w l^{2} \omega^{b}\right)=\sigma^{a}{ }_{b} w l^{2} \omega^{b} \quad \text { and } \quad \mathcal{L}_{w^{-1} u^{b}}\left(w l^{3} \omega^{a}\right)=0
$$

hold, so the vector $\epsilon w l^{3} \omega^{a}$ joins neighbouring particles (for infinitesimal $\epsilon)$, and for a (thin) vortex tube the vortex strength $w \omega \delta F$ is constant in time: $(w \omega \delta F)^{\prime}=0 .(\delta F$ is the surface area of a material section of the vortex tube orthogonal to $\left.\omega^{a}\right)$.

Corollary 1. Along the world line of a fluid particle, vorticity can neither be created or destroyed.

Corollary 2. The direction of the local rotation axis is constant (in the sense of Fermi propagation) along a flow line, if and only if it lies in a principal shear direction.

Secondly, we turn to rigid flows. For them there holds

Theorem 2.4.3. In a rigid flow, $u^{a}$ is constant along the vortex lines and $R_{a b} u^{a} u^{b}+\frac{1}{2} R+3 \omega^{2}$ is constant along the flow lines. If furthermore $u^{a}$ is a Ricci eigenvector, then $\omega$ is also constant along the flow lines. If finally (44) holds and $\omega \neq 0$, then the flow is isometric [10].

Proof. Let $\Theta=\sigma=0$. Then it follows from (21),(20) and (8) that $u^{a}{ }_{; b} \omega^{b}=0$, thus the first statement. From (37) and the definitions in subsection 2.1 there follows

$$
q^{a} \equiv h^{a b} R_{b c} u^{c}=\omega^{a b}{ }_{; b}+\omega^{a b} \dot{u}_{b}-2 \omega^{2} u^{a}
$$

and from this

$$
q^{a} ; a+\dot{u}_{a} q^{a}=-2\left(\omega^{2}\right)^{\cdot}-\omega^{a b} \dot{u}_{a ; b} .
$$

Thus through (42) (with $l=1$ since $\Theta=0$ )

$$
q_{; a}^{a}+\dot{u}_{a} q^{a}=-3\left(\omega^{2}\right)^{\circ}
$$

To prove the second statement, we need in addition the "conservation theorem" for the Einstein tensor $G_{a b} \equiv R_{a b}-\frac{1}{2} R g_{a b}$. Through $\Theta=\sigma=0$ and (21) there holds $0=u_{a} G_{; b}^{a b}=\left(u_{a} G^{a b}\right)_{; b}+G^{a b} \dot{u}_{a} u_{b}$. On the other hand by definition $G^{a b} u_{b}=q^{a}-u^{a} G_{b c} u^{b} u^{c}$, and consequently

$$
q_{; a}^{a}-\dot{u}_{a} q^{a}=\left(G_{a b} u^{a} u^{b}\right)^{\circ} .
$$

Subtracting (51) from this, one obtains the second statement of the theorem. The third result comes from (51), since $q^{a}=0$ means just that $R_{b}^{a} u^{b}$ is proportional to $u^{a}$. Finally let (44) also be true. Then (49) shows that

$$
\omega^{a b}{ }_{; b}-\omega^{a b}(\log w)_{, b}-2 \omega^{2} u^{a}=0
$$


holds. Because $\dot{\omega}=0$, the divergence of this gives that $\omega^{a b}{ }_{; a}(\log w)_{, b}=0$. Contracting (53) with $(\log w)_{, a}$ thus gives $\omega^{2} \dot{w}=0$, so that when $\omega \neq 0$ necessarily $\dot{w}=0$ must hold. However then according to (44), $\dot{u}_{a}$ is a gradient, so that according to vi. in subsection 2.2 an isometric flow occurs.

This theorem contains the noteworthy result that a rigid body in a special Einstein space $\left(R_{a b}=0\right)$ cannot change its angular velocity. This was early noticed in the case of special relativity, and was used as an argument against the validity of the concept of a rigid body in special relativity theory.

In the next theorem we will characterise the isometric flows, formally defined in vi. in subsection 2.2 , by transparent kinematic properties.

Theorem 2.4.4. The isometric flows are characterised amongst the rigid flows by the fact that in them the vorticity vector is Fermi-transported along the flow lines, and the acceleration vector always points at the same neighbouring particles:

$$
\Theta=\sigma=0: \quad \dot{u}_{[a ; b]}=0 \Leftrightarrow\left\{h_{b}^{a} \dot{\omega}^{b}=0, \quad h_{b}^{a} \ddot{u}^{b}=\omega^{a}{ }_{b} \dot{u}^{b}\right\} .
$$

Proof. From (40), for $\Theta=\sigma=0$, the first of the two equivalence statements follows:

$$
\begin{aligned}
& h_{b}^{a} \dot{\omega}^{b}=0 \Leftrightarrow u_{[a} \dot{u}_{b, c]}=0 \\
& \quad \Leftrightarrow\left\{\dot{u}_{[a, b]}=u_{[a} p_{b]} \quad \text { with } p_{a}=h_{a}^{b} \ddot{u}_{b}-\omega_{a b} \dot{u}^{b} .\right\}
\end{aligned}
$$

The second equivalence is correct, since both equations state that $\dot{u}_{[a, b]}$ is a simple bivector containing $u_{a}$. The value of $p_{a}$ follows through contraction of the equation $\dot{u}_{[a, b]}=u_{[a} p_{b]}$ by $u^{b}$ and use of the orthogonality relation $u_{a} p^{a}=0$ and the identities $\dot{u}_{a} u^{a}=0$ and (21). One can read off the correctness of the statement (54) from (55).

Theorem 2.4.4 contains a transparent and purely kinematic (i.e. independent of the field equations) characterisation of stationary and in particular static space-times. A universe is stationary, exactly when it is possible that (at least) one cloud of test-particles can move rigidly and with constant angular velocity under the influence of inner non-gravitational forces. For a test-particle with rest-mass $m$ moves according to the equation $m \dot{u}^{a}=K^{a}\left(u_{a} K^{a}=0\right)$, and we can assume that in rigid motion the magnitude of the resultant force exerted on a particle by the other particles is constant and its direction with respect to the neighbouring particles does not alter. Static universes are characterised by the fact that, in them, test-body motions of the above described kind are possible without rotation. 
Theorem 2.4.5. The trajectories of an isometric flow are Ricci eigenlines precisely when there exists a scalar $V$ such that

$$
\omega_{a}=e^{-2 U} V_{, a} .
$$

( $U$ is defined by (30).) When that occurs, $V$ obeys the equation

$$
\left(e^{-4 U} V^{; a}\right)_{; a}=0
$$

Proof. From the definitions of the kinematic quantities there follows the identity $h_{b}^{a} \omega^{b c}{ }_{i c}+\omega^{a b} \dot{u}_{b}=-\eta^{a b c d} u_{b}\left(\omega_{c d}-2 \omega_{c} \dot{u}_{d}\right)$, so we can conclude from (37) that for $\Theta=\sigma=0$

$$
u_{[a} R_{b] c} u^{c}=0 \Leftrightarrow u_{[a} \omega_{b, c]}+2 u_{[a} \omega_{b} \dot{u}_{c]}=0 .
$$

When we contract the equation on the right with $u^{a}$, use the supposition $\dot{u}_{a}=U_{, a}$ and the relation $h_{b}^{a} \dot{\omega}^{b}=0$ that follows from this according to Theorem 2.4.4, and take into account $\omega_{a} u^{a}=0$, we obtain from (58) for isometric flows the stronger equivalence

$$
u_{[a} R_{b] c} u^{c}=0 \Leftrightarrow \omega_{[a ; b]}+2 \omega_{[a} U_{, b]}=0 \Leftrightarrow\left(e^{2 U} \omega_{[a}\right)_{, b]}=0
$$

and thus the first statement of the theorem. The second statement follows directly from (56), (43) and $\dot{u}_{a}=U_{, a}$.

To conclude these remarks we prove a further theorem-in analogy with certain theorems from the theory of pure gravitational radiation fields ${ }^{17}$-which shows that curve families with specified kinematic properties in general can only exist in space-times whose conformal curvature tensor belongs to a particular Petrov type.

Theorem 2.4.6. In a space-time $W$ let $K$ be a congruence of vortex- and shear-free curves. If furthermore $K$ is rigid or $u^{a}$ is a Ricci eigenvector, then the conformal curvature tensor is of type $I$ and has real eigenvalues, and $u^{a}$ is a Weyl principal vector, or $W$ is conformally flat.

Proof. Let $\sigma=\Theta=0$. Then a glance at (35) shows that

$$
u_{[a} R_{b c] d \varepsilon} u^{e}=0
$$

and (37) reduces to

$$
u_{[a} R_{b]_{\varepsilon}} u^{e}=0
$$

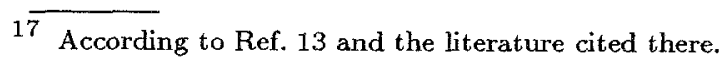


From the equation defining the conformal curvature tensor $C_{a b c d}$ and the two previous relations comes

$$
u_{[a} C_{b c] d e} u^{e}=0 \quad \text { or } \quad-{ }^{*} C_{a b c d} u^{b} u^{d}=H_{a c}=0 .
$$

This is however characteristic of the claimed properties. If on the other hand $\sigma=\omega=0$ and (60) is supposed, then according to (37) $\Theta_{, a} \sim u_{a}$. Therefore (59) holds again, so that the proof is attained.

We remark further that under the assumptions of the last theorem, $E_{a c} \equiv C_{a b c d} u^{b} u^{d}$ can be expressed in terms of $R$ and the kinematic quantities through (36)-(38), and that the theorem generalizes a known statement about static vacuum fields $[11,12]$.

\subsection{Kinematic characterisations of cosmological models}

Among the structures $(W, K)$ consisting of a four-dimensional normal hyperbolic Riemann space $W$ and a family $K$ of timelike curves imbedded in it, those used in cosmological theory as models for the metric relations of the cosmos and the (average) motion of its matter, are characterised by high symmetry. We wish to derive them from the weakest and most transparent assumptions.

Definition. A flow $(W, K)$ is called isotropic relative to $k \in K$ if a group $G$ of isometric mappings of $W$ onto itself exists with the properties

a) The mappings of $G$ leave $k$ pointwise fixed.

b) The mappings of $G$ induce permutations of $K$. to $k$.

c) $G$ acts transitively on the directions proceeding from $k$, orthogonal

When $(W, K)$ is isotropic with respect to $k$, all tensors constructed from $g_{a b}$ and $u^{a}$ are invariant under $G$. Therefore along $k$ we must have $\omega=\sigma=\dot{u}_{a}=0$ and $\Theta^{, a} \sim u^{a}$; for otherwise preferred spatial directions would be determined by $\omega^{a}, \sigma^{a}{ }_{b}, \dot{u}^{a}, h^{a b} \Theta_{, b}$, which is contradictory to c). ${ }^{18}$

Let $(W, K)$ be isotropic with respect to all $k \in K$. Then $K$ is thus a vortex-free inertial flow and according to ii. in subsection $2.2 u_{a} d x^{a}=-d t$ for a suitable scalar $t$. By $\Theta_{, a} \sim u_{a}, \Theta=\Theta(t)$; thus the scalar $l$ introduced in (39) can be chosen so that $l=l(t)$. From a) and b) it follows that the (orthogonal to $K$ ) hypersurfaces $t=$ const are mapped into themselves by $G$. According to $c$ ) and the theorem of Schur these are hypersurfaces of constant curvature $K(t)$. Because of $\sigma=0$ they are mapped conformally

$\overline{18 \text { From } \sigma_{a}^{a}}=0, \sigma_{b}^{a}$ has at least one non-degenerate eigenvector orthogonal to $u^{a}$ when $\sigma \neq 0$. 
onto each other by $K$ [see (12)]; therefore the 'expansion radius' $l(t)$, so far only determined up to a constant factor, can be chosen so that $K=$ $\epsilon l^{-2}(\epsilon= \pm 1,0)$. Since according to the definition (5) of $h_{a b}$ the metric fundamental form $G$ is equal to $h_{a b}-\left(u_{a} d x^{a}\right)^{2}$, it follows further, on use of a coordinate system $\left(x^{a}\right)$ with $\left(x^{\nu}\right)^{v}=0, x^{4}=t$, that

$$
G=l^{2}(t) d \sigma^{2}-d t^{2}, \quad u^{a}=\delta_{4}^{a}
$$

where $d \sigma^{2}$ is the metric (depending only on $x^{\nu}$ ) of a 3 -space of constant curvature $\epsilon$. The $(W, K)$ described by $(62)$ are isotropic and furthermore homogeneous; thus there holds the

Theorem 2.5.1. $(W, K)$ is isotropic with respect to all $k \in K$ exactly when $K$ describes a vortex- and shear-free inertial flow in $W$, the orthogonal hypersurfaces $R_{t}$ to $K$ are spaces of constant curvature, and the expansion velocity is constant along $R_{t}$; a normal form for $\left(g_{a b}, u^{a}\right)$ is (62). In an isotropic flow the curves of $K$ are Ricci eigenlines.

In the literature the models (62) are usually derived, following Robertson [14], from the following postulates: i) $\dot{u}=0$, ii) $\omega=0$, ii) isotropy, iv) homogeneity. Occasionally it is hinted that iv) is superfluous. Our proof displayed above shows that i) and ii), and hence the existence of a cosmic time ('Weyl's Postulate', according to Refs. 14 and 15), also follow from iii), and without any assistance from group theory. The proof given here can be regarded as the completion of the sketchily presented considerations of Einstein [16].

It should still be remarked that an isotropic flow is conformal, since $u_{a}=-t_{, a}, \sigma=0$ and $l=l(t)$ (according to $\mathrm{v}$. in subsection 2.2), with $l u^{a}$ as generating vector, so that by viii. in subsection $2.2, \lambda / l$ is constant along a light ray, and thus by (9),(10) respectively according to (34),(38) the local relative velocity, respectively relative acceleration field are given by

$$
v^{a}=\frac{i}{l} \delta_{\perp} x^{a}, \quad b^{a}=\frac{\ddot{l}}{l} \delta_{\perp} x^{a} .
$$

Kinematically distinguished special cases characterised by Theorem 2.5.1 are the following models:

a) Those that are static $\left(i=0 \Rightarrow v^{a}=0\right)$, which (independently of Theorem 2.5.1) are characterised by $\dot{u}=\omega=\sigma=\Theta=0\left(\Leftrightarrow u_{a ; b}=0\right)$,

b) The de Sitter ( $\Leftrightarrow$ 'Steady State') model $\left(L \sim e^{H t}, \epsilon=0\right)$ characterised by stationary expansion, that is $\Theta \neq 0=\dot{\Theta}=\dot{K}$ ),

c) the Milne model $\left(\epsilon=-1, l \sim t \Rightarrow b^{a}=0\right)$, characterised by flatness 
of $W .^{19}$

Now we wish to characterise the simplest models with a rotating substratum.

Theorem 2.5.2. i) Suppose $(W, K)$ describes an inertial flow with the following properties: (a) In the infinitesimal spacelike neighbourhood of each substratum element, the direction of the rotation axis is constant, i.e. $\left(\omega^{a} / \omega\right)_{; b} \delta_{\perp} x^{b} \equiv 0$. (b) The rotation axis is a main shear direction. Then $\left(g_{a b}, u^{a}\right)$ can be transformed to the normal form

$$
\begin{aligned}
u^{a} & =\delta_{4}^{a} \\
G & =d z^{2}+\gamma_{A B}\left(x^{A}, t\right) d x^{A} d x^{B}-\left(d t+u_{A}\left(x^{B}\right) d x^{A}\right)^{2} \quad(A=1,2)
\end{aligned}
$$

and this normal form is characteristic of the stated properties.

ii) Suppose $(W, K)$ has the properties named in i) and also $\sigma=0$. Then $\Theta=0$, in (64) $\gamma_{A B, t}=0$, and $\omega$ is constant in the vortex surfaces.

iii) If additionally to ii) $u^{a}$ is a Ricci eigenvector, then $\omega$ is constant (and with this $\omega_{a ; b}=0$ ).

Proof. i) From $\dot{u}=0$, (b) and Theorem 2.4 .2 follows $\left(\omega^{a} / \omega\right)=0$; from this and from (a) it follows that $\left(\omega^{a} / \omega\right)_{; b}=0$. Then $W$ decomposes directly, that is $G=d z^{2}+G^{\prime}\left(x^{A}, t\right)$ with $\omega^{a}=\omega \delta_{3}^{a}\left(z \equiv x^{3}\right)$. Through $u^{a} \omega_{a}=0$, thus $u^{3}=0$, the natural projections of the flow-lines in the 3 space $\left(x^{A}, t\right)$ are geodesic with respect to $G^{\prime}$, and thus (64) can be attained. The converse is trivial.

ii) From the normal form (64) one can read off that the neighbouring particles with equal $x^{A}$ are rigidly connected, so that from $\sigma=0$ and using (12) $\Theta=0$ and from this further follows $\gamma_{A B, t}=0$. According to Theorem $2.4 .2, \omega^{a}$ is parallelly transported along the flow lines, thus $\omega_{, a} u^{a}=0$. Furthermore according to (43), $0=\left(\omega^{a} / \omega\right)_{; a}=\omega_{, a} \omega^{a}$. Consequently $\omega=\omega\left(x^{A}\right)$.

iii) According to Theorem 2.4.5 now $\omega_{a}=\omega z_{a}$ is a gradient, thus $\omega=\omega(z)$. On the other hand $\omega=\omega\left(x^{A}\right)$; the two together give $\omega=$ const.

We will use this theorem later to make possible a simple derivation of the Gödel cosmological model.

In the case of rigid inertial flows through $(9),(10)$ and $(34),(38)$ the relative velocity and acceleration are given by the relations

$$
v^{a}=\omega^{a}{ }_{b} \delta_{\perp} x^{b}, \quad b^{a}=\omega^{a} \omega_{b} \delta_{\perp} x^{c}-\omega^{2} \delta_{\perp} x^{a}
$$

\footnotetext{
$\sqrt{19 R_{a b}=0}$ is sufficient, since all models $(62)$ are conformally flat because of the existence of a three-dimensional isotropy group, see e.g. Ref. 12, p.43.
} 
of elementary kinematics. According to viii. in subsection 2.2 no change of the frequency of light signals occurs in such motions (even in the case of distant particles). The transversal Doppler effect is here compensated by the gravitational 'redshift'. (In the Minkowski universe rigid rotational flows are necessarily non-geodesic, so that such a compensation cannot take place, cf. (36).

\section{DYNAMICS}

\subsection{The Gravitational Field equations. Similarity law.}

The local equivalence of inertial and gravitational forces, empirically proved with great accuracy, but only formulisable through an ad hoc hypothesis in the framework of the conceptual system of Newtonian mechanics and gravitational theory, led Einstein to the supposition that both these "forces" are expressions of one and the same structural property of the space-time manifold, for which Weyl coined the expression guiding field. The local validity of the special theory of relativity makes necessary that this structure is mathematically represented by a normal hyperbolic Riemannian metric. ${ }^{20}$

Since on the one hand matter generates the gravitational field and the Poisson equation describes well what is experienced, and on the other hand in the new theory $g_{a b}$ are the state variables for the guiding field, and since in special relativity theory the mechanical properties of matter are described through the energy-momentum-pressure tensor $T_{a b}$, it is a reasonable assumption that

(1) The components $T_{a b}$ of the matter tensor are equal to quasi-linear differential expressions of the second order in the components $g_{a b}$ of the metric tensor.

According to a theorem proved by Weyl (Ref. 18, Appendix II), it follows from this very general assumption alone that the field equations have the form ${ }^{21}$

$$
G_{a b}+(M G-\Lambda) g_{a b}=-\kappa T_{a b}
$$

where $\Lambda, M$ and $\kappa(\neq 0)$ are constants with dimensions ${ }^{22}-2,0,0$ respectively.

This equation is non-linear in the $g_{a b}$, that is the assumption (1) already contains that the metric field mediates an interaction between matter-filled world-tubes.

20 The presentation of the foundations of Einstein's gravitational theory given here follows-with some modification and supplementation-Refs. 18 and 19.

$21 G_{a b} \equiv$ Einstein Tensor, $G \equiv G^{a}{ }_{a}$; see our conventions given above.

22 With respect to length; $c \equiv 1, \gamma \equiv \frac{1}{8 \pi}$, according to our conventions. 
The symmetry of the matter tensor does not have to be introduced here as an independent hypothesis (equivalence of momentum- and energydensity) as in special relativity, rather it follows-if one considers postulate 1) as an implicit definition of $T_{a b}$-from the Weyl Theorem mentioned above: namely there exists in a Riemann space no quasi-linear, invariant bivector of 2nd order in the metric $g_{a b}$. In General Relativity theory, the symmetry of the matter tensor is a necessary condition, in order that it can occur as a source of the metric field in the sense of (1).

From (66) it follows that $-G(1+4 M)+4 \Lambda=\kappa T$. Therefore since $T=$ const. is not always true, $1+4 M \neq 0$. From this one obtains from (66) on using the Bianchi identities the divergence equation

$$
T_{a ; b}^{b}=e T_{, a} \quad\left(e \equiv \frac{M}{1+4 M}\right) .
$$

For the particularly simple case of matter only interacting through gravity, with its proper rest mass density of $\rho$ and $u^{a}$ its four-velocity, according to the fundamental ideas of the theory sketched above one can set (see subsections 3.4 and 3.5 )

$$
T_{a b}=\rho u_{a} u_{b} \quad(\rho>0) .
$$

Equations (67) is then equivalent to the system of equations

$$
\left(\rho u^{a}\right)_{; a}=e \dot{\rho}, \quad \dot{u}_{a}=-e h_{a}^{b}(\log \rho)_{, b} .
$$

The assumptions introduced this far thus lead to a theory in which incoherent matter moves on the geodesics of the metric $\bar{g}_{a b}=\rho^{2 e} g_{a b}$ (Lemma $2.4)$ and in which spontaneous rest-mass creation takes place with the production density $e \dot{\rho}=(e / e-1) \rho \Theta$.

If one introduces either the additional assumption (2a) The rest mass of matter that interacts only by gravity is conserved, or

(2b) The flow lines of matter that interacts only through gravity are geodesics with respect to the 'natural' metric $g_{a b}$,

it follows that $e=M=0$, and we obtain from (66) the Einstein Field Equation

$$
G_{a b}-\Lambda g_{a b}=-\kappa T_{a b}
$$

and from this the so-called conservation theorem

$$
T_{; b}^{a b}=0
$$


that for (68) again leads to (2a) and (2b) as consequences.

Since at the present time there exists no reason to reject the simple assumption (2a) and independently, the hypothesis (2b) fulfils the idea of the guiding field in the simplest manner [26], we take as a basis in the further development of this work the classic equation (70).

The more general theory with $e \neq 0$ can easily be developed and leads for $|e| \ll 1$ to a theory that is similar to Jordan's 'extended gravitational theory' (Ref. 25, books 2,3) and to Hoyle's cosmological theory (Refs. 15,27 and the works cited there), but is simpler and, it seems to us, better grounded from the viewpoint of local physics (see above); we introduce no new hypothesis, rather omitting only one of the axioms of the Einstein theory, and indeed the mathematically least important one. It is easy to see that this theory is incompatible with a stationary state in the sense of the PCP of Bondi and Gold [26]. We remark further that in the theory with $e \neq 0,(71)$ holds for a Maxwell field, so that the theory of light propagation remains unaltered.

Through consideration of the spherically symmetric solutions ${ }^{23}$ of (70), it turns out (with our choice of units) that

$$
\kappa=1
$$

must be supposed. The positivity of $\kappa$ expresses the fact that matter attracts gravitationally rather than repels.

The cosmological term $\Lambda g_{a b}$ in (70) can be excluded $(\Lambda=0)$ through either of the following two assumptions:

(3a) Apart from the speed of light and the Newtonian gravitational constant, the field equation contains no dimensional constants.

(3b) In a vacuum, the trace of the tensor which transforms the relative position vector of neighbouring free particles into their relative acceleration has vanishing trace ${ }^{24}$ (corresponding to the Laplace equation of Newtonian Theory).

Although we regard the specialisation $\Lambda=0$ as appropriate on methodological grounds as long as experience does not indicate $\Lambda \neq 0$, for mathematical generality we will set $\Lambda=0$ only when this restriction permits derivation of theorems that otherwise would not be valid.

23 Or through (82) or alternatively (123), where to begin with $\kappa$ is retained and then its value determined through correspondence with the corresponding equations of Newtonian Theory.

24 See (34) for $\dot{u}=0$. (3b) is Pirani's characterisation of special Einstein spaces, see [28]. 
According to the laws of classical hydrodynamics (with gravitational interaction) for every flow there exists a two-parameter family of similar flows. This is closely connected to the fact that in the basic equations there enters only one universal constant with dimensions, the gravitational constant, so that only one of the three basic dimensions of length, mass and time (let us say the mass) can be eliminated by a 'rational' choice of units. In relativity theory, already kinematics contains a constant, namely $c$; one can thus expect a one-parameter family of similar flows to a given flow for $\Lambda=0$, and none for $\Lambda \neq 0$.

In fact the field equation (70) shows that for $\Lambda=0$ (and only then) out of each solution $\left(g_{a b}, T_{a b}\right)$ a family of similar solutions $\left(\bar{g}_{a b}, \bar{T}_{a b}\right)$ can be obtained through $\bar{g}_{a b}=m^{2} g_{a b}, \bar{T}_{a b}=m^{2} T_{a b},(m>0)$. The space-time $\bar{W}$ is equivalent to $W$, only if $W$ admits a homothetic mapping onto itself.

In a classification of exact solutions of (70) with $\Lambda=0$ it is useful to arrange the solutions into families that are similar to each other, and to choose as characterising data for a particular solution a 'similarity parameter' (proportional to the above $m$ ), together with such invariants or properties as characterise the whole family. ${ }^{25}$

For example within the Schwarzschild family of vacuum solutions the gravitational radius is such a similarity parameter, and the family as such is characterised by the property of 'spherical symmetry'. Examples of when the above similarity transformation leads to equivalent worlds are provided by the Levi-Civita static cylindrically symmetric vacuum fields.

\subsection{Conservation of rest mass, energy theorem and equation of motion for ponderable matter}

To determine if and in what sense the divergence equation (71) also describes the conservation of energy and momentum in gravitational fields $\left(R_{a b c d} \neq 0\right)$, one can either convert it (or the corresponding equation for the tensor density $(-g)^{1 / 2} T^{a b}$ ) into an ordinary divergence equation by addition of an affine tensor and thus set up for finite volumes two local conservation balances containing as well as material also gravitational energy and momentum - which as is known, on many grounds has not yet led to clear insights-or introduce an average four-velocity $u^{a}$ for the matter and set up substantial balances related to matter elements. We will briefly describe the second procedure, corresponding to thermo-hydrodynamics. ${ }^{26}$

25 To the latter belong for instance the Petrov type of the conformal tensor and the structure tensor of an isometry group.

26 We follow essentially Eckart [20], whose results we complete through the 'NavierStokes' equation (76). 
First one can uniquely decompose a symmetric tensor field $T_{a b}$ with respect to an arbitrary timelike unit vector $u^{a}$ thus:

$$
T_{a b}=\mu u_{a} u_{b}+p h_{a b}+2 u_{(a} q_{b)}+\pi_{a b}
$$

with $h_{a b}$ according to (5), and

$$
q_{a} u^{a}=0, \quad \pi_{a b} u^{b}=0, \quad \pi_{a}^{a}=0 .
$$

By contraction of (71) with $u_{a}$, partial differentiation and using the kinematic quantities (defined in 1.1) formed from $u_{a}$, one can derive the equation

$$
\dot{\mu}+(\mu+p) \Theta+\pi_{a b} \sigma^{a b}+q_{; a}^{a}+q_{a} \dot{u}^{a}=0 .
$$

Correspondingly the 'spacelike' part of the equation (71) gives

$$
(\mu+p) \dot{u}_{a}+h_{a}^{b}\left(\dot{q}_{b}+p_{, b}+\pi_{b ; c}^{c}\right)+\left(\omega_{a b}+\sigma_{a b}\right) q^{b}+\frac{4}{3} \Theta q_{a}=0 .
$$

This pair of equations is, for a given decomposition (73), equivalent to the relation (71).

The equations (70) and (74),(75) attain a concrete physical meaning only when the nature of the matter generating the field is stated and the introduced auxiliary quantities are subject to corresponding equations of state.

For bulk matter it is permissible to assume that for a uniquely determined 4-velocity ${ }^{27} u^{a}$, the main part of the energy density $\mu$ is the positive rest-mass proper density $\rho$ that remains conserved,

$$
\left(\rho u^{a}\right)_{; a}=0,\left(\Leftrightarrow \rho \dot{v}=\Theta, \quad v \equiv \frac{1}{\rho}=\text { specific volume }\right) .
$$

The quantity $u=\mu / \rho$ (which in general differs but little from 1 ) is the specific internal energy. Under this assumption we can designate the relation [following from $(74),(76)$ ]

$$
\rho \frac{d u+p d v}{d s}+q_{; a}^{a}+\pi_{a b} \sigma^{a b}+q_{a} \dot{u}^{a}=0
$$

as the substantial thermodynamic energy balance, the equation (76) as the momentum balance or equation of motion, ${ }^{28}$ and the quantities $p, q^{a}$ and

\footnotetext{
27 The local 'barycentric' velocity, see e.g. [21].

28 Lichnerowicz [2] has, through use of the auxiliary metric referred to in Lemma 2.4, proposed another relativistic equation of motion which we consider as physically unfounded.
} 
$\pi_{a b}$ respectively as the pressure, energy-flow density relative to the matter (arising from transport processes such as diffusion and heat conduction), and viscous pressure tensor.

These equations differ from the corresponding non-relativistic ones only through the very small terms $q_{a} \dot{u}^{a}$ respectively $h_{a}^{b} \dot{q}_{b}+\left(\omega_{a b}+\sigma_{a b}+\right.$ $\left.\frac{4}{3} \Theta h_{a b}\right) q^{b}$, necessitated by the inertia of energy, and through the fact that in $(76),(\mu+p)$ occurs as the effective density of the inertial mass instead of $\rho$.

From the definition (39) of $l$ and (77) it follows that the conservation of rest mass can also be written in the particularly transparent form

$$
\rho l^{3}=\text { const. (along the flowlines). }
$$

From the definition of the Einstein tensor, (70),(73) and (74) follows

$$
R=3 p-\mu-4 \Lambda, \quad R_{a b} u^{a} u^{b}=\Lambda-\frac{1}{2}(\mu+3 p), \quad h^{a b} R_{b c} u^{c}=q^{a} .
$$

Thus the matter flow-lines are Ricci eigenlines, exactly when there is no energy flow relative to the matter.

The last remark and (80) permit a dynamic interpretation of a statement in subsections 1.4 and 1.5 .

From (36),(39) and (80) results the equation

$$
3 \frac{\ddot{l}}{l}+2\left(\sigma^{2}-\omega^{2}\right)-\dot{u}_{; a}^{a}+\frac{1}{2}(\mu+3 p)-\Lambda=0 .
$$

This shows that in rigid motions, the magnitude $-\Lambda+\frac{1}{2}(\mu+3 p)-2 \omega^{2}$ is the source density of the acceleration field. This statement can be interpreted as the relativistic analogue of the Gauss theorem $\operatorname{div} \mathbf{F}=-\frac{1}{2} \rho+2 \omega^{2}(\mathbf{F} \equiv$ the field strength of the gravitational and centrifugal fields in a rigidly rotating system). ${ }^{29}$ For isometric flows one obtains via (30) the 'Poisson' equation

$$
U_{a}^{; a}=-\Lambda+\frac{1}{2}(\mu+3 p)-2 \omega^{2} \quad(\dot{U}=0) .
$$

Reformulating this as a three-dimensional divergence equation suggests designation of $(\mu+3 p)$ as the effective density of the active gravitational mass [28].

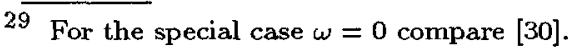




\subsection{Entropy Theorem. Ideal fluids. Isentropic flows.}

An exact description of matter requires in addition to the concepts and laws introduced in the previous section further thermodynamic considerations, that will not be carried out here in full generality; for the relativistic thermodynamics of irreversible processes we refer to the beautiful Handbuch article by Meixner and Reik [21], the literature cited there, and to Just [22].

We will only sketch one example in anticipation of the next section, and on account of the soon to be handled case of an ideal fluid: the one component fluid (following Ref. 20).

For such a fluid we require first the existence of a (thermal) equation of state

$$
u=u(p, v) \text {. }
$$

Consequently there exist (scalar) functions $T(p, v)$ and $s(p, v)$ such that

$$
d u+p d v=T d s
$$

With this, on referring back to (77) we can transform the energy equation (78) into

$$
\begin{aligned}
\rho \dot{s}+\left(\frac{q^{a}}{T}\right)_{; a} & =\left(\rho s u^{a}+\frac{q^{a}}{T}\right)_{; a}=\eta \\
\eta & \equiv-\frac{1}{T}\left(\pi_{a b} \sigma^{a b}+q_{a}\left(\dot{u}^{a}+(\log T)^{, a}\right)\right) .
\end{aligned}
$$

Under the further assumption $T>0$ it is suggested to interpret $T$ as the temperature, $s$ as the specific entropy, and this equation as the entropy balance equation, which-in agreement with the meaning of $\pi_{a b}, \sigma_{a b}$ [see (12)], $q^{a}$ and $T$-leads to the phenomenological equations

$$
\begin{aligned}
\pi_{a b} & =-\lambda \sigma_{a b} & & (\lambda(p, v) \equiv \text { viscosity coefficient }) \\
q_{a} & =-\kappa h_{a}^{b}\left(T_{, b}+T \dot{u}_{b}\right) & & (\kappa(p, v) \equiv \text { heat conduction coefficient })
\end{aligned}
$$

The entropy theorem demands that the entropy production density $\eta$ is non-negative, and consequently

$$
\lambda \geq 0, \quad \kappa \geq 0
$$

must be true. The 4-vector

$$
S^{a} \equiv \rho s u^{a}+\frac{q^{a}}{T}
$$


is to be designated as the entropy flow density. It appears in (89) separated into a convection part $S^{a} \equiv \rho s u^{a}$ and a conduction part $q^{a} / T$, and always has non-negative divergence.

In the equations (75),(76) the terms containing $\pi_{a b}$ and $q^{a}$ are often very small in relation to the others; consequently one can then, in particular when studying the interaction of matter with the gravitational field (taken into account implicitly through the covariant derivatives), ignore these terms. This leads to the introduction of the model of an ideal fluid defined in view of $(86),(87)$ by

$$
\lambda=0, \quad \kappa=0 .
$$

For an ideal fluid therefore according to (73) and (5),

$$
T_{a b}=\mu u_{a} u_{b}+p h_{a b}=(\mu+p) u_{a} u_{b}+p g_{a b} .
$$

(Since $-u^{a} u_{b}$ and $h_{b}^{a}$ are projections orthogonal to each other, the first equation (91) is the spectral decomposition of $T_{b}^{a}$.)

The energy and momentum laws simplify to

$$
\begin{gathered}
\dot{u}+p \dot{v}=0, \\
(\mu+p)+h_{a}^{b} p_{, b}=0,
\end{gathered}
$$

and for a chemically homogeneous ideal fluid ${ }^{30}$ according to (94), always

$$
s=\text { const. along the flow lines; }
$$

all processes in them proceed (adiabatically) reversibly. The flow-lines are Ricci eigenlines, by (91), (77).

According to the rules of thermodynamics, the specific enthalpy

$$
w=w(s, p)=u+p v=\frac{\mu+p}{\rho}
$$

can be used as a thermodynamic potential; through elimination of $s$ by use of the equations

$$
\frac{\partial w}{\partial s}=T, \quad \frac{\partial w}{\partial p}=v
$$

30 During chemical reactions (84) must be replaced by the Gibbs fundamental equation, and in general (94) is no longer valid. 
one obtains the thermal equation of state $T=T(p, v)$ and further the caloric equation of state (83). According to (95) and (96) along an isentrope

$$
w(s, p)=w\left(s, p_{0}\right) \exp \left(\int_{p_{0}}^{p} \frac{d p}{\mu+p}\right) .
$$

A flow is called isentropic when, even in the whole domain of the flow, $s=$ const. (compare Ref. 4 as well as Ref. 38). In an isentropic flow, $\mu, p$ and $\rho$ are pairwise functionally dependent, and according to (97) and (95)

$$
\frac{d w}{w}=\frac{d p}{\mu+p}, \quad \frac{d p}{d w}=\frac{\mu+p}{w}=\rho .
$$

The equation of motion (93) is thus equivalent to

$$
\dot{u}_{a}=-h_{a}^{b}(\log w)_{, b}
$$

[eq. (44) above]. From this we can extract

Theorem 3.3.1. In an isentropic flow of a one-component ideal fluid, the pressure is a function of the specific enthalpy. The matter tensor, rest mass density and specific energy are determined by the formulae

$$
T_{a b}=\rho w u_{a} u_{b}+p g_{a b}, \quad \rho=\frac{d p}{d w}, \quad u=w-\frac{p}{\rho}
$$

(with $w>0,(d p / d w)>0)$. The divergence of $T_{a b}$ vanishes precisely when $\left(\rho u^{a}\right)_{; a}=0$ and the streamlines are geodesics ${ }^{31}$ of the auxiliary metric $\bar{g}_{a b}=w^{2} g_{a b}$. The energy theorem (92) is identically fulfilled as a consequence of the last two equations (99).

Instead of the variables $\left(w, u^{a}\right)$ and the function $p(w)$ one could also take $\left(p, u^{a}\right)$ with $\mu(p)$ (e.g. Refs. 3,2$)$ or with $\rho(p)$ (e.g. Refs. 19,20); however the representation in the theorem is the simplest because of (44). We mention also the often used (e.g. Refs. 3,2) representation following from (98),(99):

$$
T_{a b}=\rho\left(\stackrel{a}{w}+\int_{p_{0}}^{p} \frac{d p}{\rho}\right) u_{a} u_{b}+p g_{a b} \quad\left(\stackrel{0}{w} \equiv w\left(p_{0}\right), \rho=\rho(p)\right)
$$

31 Eisenhart [24] recognised this geodesy in another way. Through the formal analogy between (45) and the Fermat principle of geometric optics, Synge [3] designated $w$, whose thermodynamic meaning he did not mention, as the 'index' of the fluid. 
in which usually the unnecessary and often impossible specialisation $p_{0}=$ $0, w=1$ is presupposed.

According to Theorem 2.4.1 and (47) the flow- and vortex-lines span vortex surfaces [2], and from Theorem 2.4.2 there follows for isentropic flows the dynamic vortex theorem (48) (with the present meaning for $w$ ); (97) shows the influence of the pressure on the vorticity.

In an isentropic flow, $\Theta=0$ has the consequence that $w$ is constant along the flow lines, so that with (44) and according to Lemma 2.2.6 there holds the following theorem.

Theorem 3.3.2. A rigid isentropic flow is only possible in a stationary gravitational field [4]. Then $\xi^{a}=u^{a} / w$ is a Killing vector and $U=-\log w$ the scalar gravitational potential [defined in 2.2.6 and occurring in (82)]. The vortex vector $\omega^{a}$ is (Fermi-) constant along the flow lines (Theorem 2.4.1) and satisfies the equations (56),(57).

In conclusion we remark that according to Theorem 2.4.6, a vortexand shear-free isentropic flow is only possible in a gravitational field whose conformal tensor is of Type I and has real eigenvalues. This corresponds to the conception that such fields contain no gravitational waves, which are apparently always tied in with shearing motions of the matter that interacts with the field (according to Refs. 31 and 32, sec.2-4.8).

\subsection{Kinetic gas theory. H-Theorem. Equilibrium distributions in a gravitational field.}

In this section we will not idealise the matter from the beginning as a continuum, but rather describe it through the model of a statistical assembly of particles of rest mass $m$ that only interact with each other through elastic collisions. We take it that the space-time in a "physically infinitely small region' can be regarded as flat, as is required for the definition of the moments of the distribution function and in particular for the formulation of the Boltzmann collision equation, although the 'gas' as a whole may find itself in a gravitational field that then (expressed in analogy to electron theory) can be regarded as the 'macroscopic' field.

To define the (one-particle) distribution function $F(x, p)^{32}$ we think of an infinitesimal space element characterised by the (pseudo-) vector $d \vec{x}_{a}$ at the (arbitrary) event $x$, and in momentum space ${ }^{33}$ an infinitesimal cell at the point $p^{a}$ on the mass-hyperboloid

$$
p_{a} p^{a}=-m^{2}
$$

\footnotetext{
32 We leave out the indices of the arguments: $p \equiv\left(p^{a}\right)$ and so on. 
to which then analogously a $d \dot{p}_{a}$ belongs. $F$ is defined by the statement that

$$
F\left(x^{a}, p^{a}\right)\left|d \dot{x}_{a} d \dot{p}^{a}\right|
$$

is the number of those particles whose worldlines intersect the space- element $d \vec{x}_{a}$ and whose momentum lies in the cell $d \ddot{p}_{a}$. Clearly $F$ is a scalar.

The function $F$ obeys the relativistic Boltzmann equation formulated by Sasaki (Ref. 33; see also Ref. 37)

$$
p^{a} F_{, a}=\iiint\left(F^{\prime \prime} F^{\prime \prime \prime}-F F^{\prime}\right) W\left(p, p^{\prime} ; p^{\prime \prime}, p^{i \prime \prime}\right) d P^{\prime} d P^{\prime \prime} d P^{\prime \prime \prime} .
$$

The partial derivative $F_{,}$, is to be thought of as relative to $x^{a}$ with parallelly propagated $p^{a}$. The first argument in $F^{\prime \prime}, F^{\prime \prime \prime}, F, F^{\prime}$ is always $x$, the second respectively $p^{\prime \prime}, p^{\prime \prime \prime}, p, p^{\prime} ;$ furthermore we set

$$
d \dot{p}_{a} \equiv p_{a} d P
$$

and so on. $W\left(p, p^{\prime} ; p^{\prime \prime}, p^{\prime \prime \prime}\right)$ describes the probability for collisions $p, p^{\prime} \rightarrow$ $p^{\prime \prime}, p^{\prime \prime \prime}$ (with corresponding phase space ranges). We have assumed

$$
W\left(p, p^{\prime} ; p^{\prime \prime}, p^{\prime \prime \prime}\right)=W\left(p^{\prime}, p ; p^{\prime \prime \prime}, p^{\prime \prime}\right)=W\left(p^{\prime \prime}, p^{\prime \prime \prime} ; p, p^{\prime}\right) .
$$

For the first moment

$$
g^{a}(x) \equiv \int g(p) p^{a} F(x, p) d P
$$

of an arbitrary function $g(p)$ there follows from (103),(105),

$$
\begin{aligned}
g_{; a}^{a}= & \frac{1}{4} \iiint \int\left(g+g^{\prime}-g^{\prime \prime}-g^{\prime \prime \prime}\right) \times \\
& \times\left(F^{\prime \prime} F^{\prime \prime \prime}-F F^{\prime}\right) W d P d P^{\prime} d P^{\prime \prime} d P^{\prime \prime \prime} .
\end{aligned}
$$

This balance equation holds analogously also for tensors $g^{a b \ldots}$ instead of $g$.

When $g$ is an additive collision invariant, there holds therefore $g_{; a}^{a}=$ 0 . From this follows:

(i) The material flow density

$$
\rho u^{a} \equiv \int m p^{a} F d P \quad\left(u_{a} u^{a}=-1\right)
$$


$(g=m)$ obeys the continuity equation

$$
\left(\rho u^{a}\right)_{; a}=0 .
$$

( $\rho$ is according to $(102),(104),(108)$ the density of the restmass in the local rest system determined by the four-velocity $u^{a}$.)

(ii) The matter tensor

$$
T^{a b}=\int p^{a} p^{b} F d P
$$

$\left(g \rightarrow p^{a}\right)$ is divergence-free:

$$
T_{; b}^{a b}=0 .
$$

Next we define through

$$
S^{a} \equiv-\int p^{a} F \log F d P
$$

the entropy flow density of our gas. There holds

$$
S_{; a}^{a}=-\int(1+\log F) F_{, a} p^{a} d P
$$

from which by the same transformation that leads to (107),

$$
S^{a}{ }_{; a}=\frac{1}{4} \iiint \int \log \left(\frac{F^{\prime \prime} F^{\prime \prime \prime}}{F F^{\prime}}\right)\left(F^{\prime \prime} F^{\prime \prime \prime}-F F^{\prime}\right) W d P d P^{\prime} d P^{\prime \prime} d P^{\prime \prime \prime}
$$

arises. Thus there holds the following covariant relativistic form of the Boltzmann H-Theorem:

Theorem 3.4.1. The entropy production density

$$
S^{a} ; a \equiv \eta
$$

is non-negative and only vanishes in a world region if there at every point $F$ is an additive collision invariant. ${ }^{34}$

If on the grounds of the relativistic collision laws we make the very plausible assumption that (corresponding to the Grad theorem [34] in the

34 Therefore in special relativity the 'total entropy at a fixed time' is only a scalar when an equilibrium distribution is at hand. 
non-relativistic theory) the general collision invariant has the form $c-\xi_{a} p^{a}$, it follows from Theorem 3.4.1 that the general equilibrium distribution of a relativistic gas has the form

$$
\stackrel{\circ}{F}(x, p)=C(x) \exp \left(\xi_{a}(x) p^{a}\right) .
$$

From this and (108),(109) there follows

Theorem 3.4.2. (Ref. 35, sec.14, where. further references are given.) For local equilibrium, the distribution function of an ideal gas has the form

$$
\stackrel{\circ}{F}(x, p)=\frac{\rho(x) \xi}{4 \pi m^{3} K_{2}(m \xi)} \exp \left(\xi u_{a} p^{a}\right) .
$$

The corresponding matter tensor is equal to that of an ideal fluid (91) with the equation of state

$$
u=G\left(\frac{1}{p v}\right)-p v
$$

where $G(y)$ is given by

$$
G(y) \equiv \frac{2}{y}-\frac{K_{2}^{\prime}(y)}{K_{2}(y)}, \quad K(y) \equiv \int_{0}^{\infty} \exp (-y \cosh z) \cosh (2 z) d z .
$$

The partition parameter $\xi$ is related to the density and pressure by

$$
m \xi=\frac{\rho}{p} .
$$

We now determine what conditions the Boltzmann equation (103) imposes on the state variables $\rho(x), \xi(x)$ and $u^{a}(x)$.

Theorem 3.4.3. An equilibrium distribution (113) is possible only in a stationary gravitational field, and indeed $\xi^{a}=\xi u^{a}$ must be a Killing vector; thus the flow is isometric. Furthermore in the flowfield

$$
\frac{p \xi^{2} x}{K_{2}(m \xi)} \equiv A=\text { const. }
$$

must hold. These conditions are sufficient, too.

Proof. For an equilibrium distribution (112), because of four-momentum conservation at collisions, (103) reduces to the Liouville equation

$$
p^{a} \stackrel{\mathrm{o}}{F}_{, a}=0,
$$


which is thus written out with $\log C=c$ to

$$
c_{, a} p^{a}+\xi_{a ; b} p^{a} p^{b}=0 .
$$

This equation must be fulfilled at every point $x$ for all $p^{a}$ that lie in the forwards lightcone, with $p_{a} p^{a}=-m^{2}$. From this follows (for example by choosing some special values for the $p^{a}$ )

$$
c_{, a}=0, \quad \xi_{a ; b}=0,
$$

whereby with $(112),(113),(116)$ the theorem is proved, since from (120) naturally again (118) follows.

Comparison of this theorem with the corresponding theorem of the non-relativistic theory (Ref. 39 and cf. Ref. 36) shows:

The reversible flows of a relativistic ideal gas are kinematically more strongly restricted than those of a non-relativistic one; they are namely not only shear-free, but also volume-preserving. Thus, in the isotropically expanding models of relativistic cosmology, one cannot understand the substratum-as in the corresponding Newtonian models [40,41]--as an ideal gas in equilibrium. ${ }^{35}$

Next we decompose the entropy flow (110) following the example of equation (89) into a convection and a conduction part:

$$
S^{a} \equiv \rho s u^{a}+s^{a} \quad\left(u_{a} s^{a} \equiv 0\right) .
$$

For an equilibrium distribution, $s^{a}$ vanishes according to (110),(112), and one obtains an expression for $s$ after a short calculation (in the rest frame), that is connected to $u$ from (114) through $\xi(d u+p d v)=d s$. Thereby $1 / \xi \equiv T$ is identified as the temperature and the connection with the phenomenological theory of the previous section recovered-at least in the equilibrium case.

The relation $\xi=1 / T$, Theorem 3.4.3, and the remark 2.2.vi give Theorem 3.4.4. For an ideal gas in thermodynamic equilibrium in a gravitational field, there exists the relation

$$
T e^{U}=\text { const. }
$$

between temperature $T$ and the gravitational potential $U$.

35 Thereby a question posed by Heckmann, Ref. 40 p.50, is answered at least in a simple case. 
Tolman [42] derived the relation (122) for a (phenomenologically described) isotropic radiation field in a static gravitational field; for the general stationary case this is contained in Theorem 3.3.2, since for the "fluid" considered there one can take isotropic radiation, which is permitted.

It should be pointed out that in the case of a rotating gas, the centrifugal potential is also contained in $U$; the temperature difference for this case asserted by (122) is of the same order of magnitude as that recently measured by Pound and Rebka [43] through the transverse Doppler effect by means of the Mössbauer effect.

Through the use of distribution functions that differ from (113) only by such a small amount that we can still set $\log F \simeq \log \stackrel{0}{F}$, one can still through the definitions of $q^{a}[(73),(108),(109)]$ and $s^{a}[(121),(110)]$ determine that $s^{a}=(1 / T) q^{a}$. Thereby then the phenomenological entropy theorem [(85) with $\eta \geq 0]$ is recognized as a consequence of the statistical H-theorem 3.4.1. The phenomenological equations (86),(87) have been motivated statistically by Sasaki [33] (without use of the entropy concept); his equations appear to us however not to be sufficiently general.

We remark further, that the assumptions made by Synge about the direction of time (Ref. 35, p.54) in handling of shockwaves in gases, follow from the H-theorem. It should however be stressed that because of Theorem 3.4.3, the use of equations (114)-(116) in general flows is strictly speaking not permitted.

\subsection{Dynamics of incoherent matter.}

Incoherent matter is formally defined by the matter tensor

$$
T_{a b}=\rho u_{a} u_{b}
$$

One can obtain this expression if in the general representation (73) one regards the pressure $p$, the eigenvalues of the viscosity tensor $\pi_{a b}$, and the total energy-flow density $q^{a}$ as negligibly small in comparison with the energy density $\mu$ [and then sets $\mu=\rho$ through (77)]. If one takes as a foundation the statistical model of matter handled in the previous section, formulae (108),(109) or the distribution function (113) show that (68) emerges exactly when $F$ has a sharp maximum in momentum space at the point $p_{a}=m u_{a}$ (the limiting case $T \rightarrow 0$.).

In this subsection (68) will be taken as given.

Almost all the previously formulated theorems can be applied to (68); we collect some together here.

i. The streamlines are geodesic: $\dot{u}=0$.

ii. The (proper) mass density is conserved: $\rho l^{3}=M, \dot{M}=0$. 
iii. In vortex-free flows, there exists a time coordinate $t$ that is equal to proper-time along the flow lines, synchronised to be equal to each other; the hypersurfaces $t=$ const. are geodesically parallel.

iv. Conformal, non-rigid flows are vortex-free [see (28)]

v. Rigid flows are isometric: $u^{a}$ then generates a translation group, and $\omega^{a}$ is harmonic. ${ }^{36}$

vi. The usual Doppler formula $d \lambda / \lambda=(\delta l)^{\circ}$ holds for the redshift of infinitesimally neighbouring particles.

vii. Stream- and vortex-lines span (2-) surfaces [see (46)].

viii. For shear-free motion, $\left(l^{2} \omega^{a}\right)^{\cdot}=0$, thus $l^{2} \omega \equiv \Omega, \dot{\Omega}=0$. (With ii.: The (Newtonian) angular momentum of a sphere (consisting of specified matter particles) of radius $l$ is $J=(8 \pi / 15) M \Omega, \dot{J}=0$.)

ix. The flow lines are Ricci lines: [see (37)]:

$$
h_{b}^{a}\left(\omega_{; c}^{b c}-\sigma_{; c}^{b c}+\frac{2}{3} \Theta^{, b}\right)=0 .
$$

$x$. Curvature scalar, density and cosmological constant stand in the relation $R=-\rho-4 \Lambda$ [see (80)].

xi. There holds the "expansion equation" 37 [see (81)]

$$
3 \frac{\ddot{l}}{l}+2\left(\sigma^{2}-\omega^{2}\right)+\frac{1}{2} \rho-\Lambda=0 .
$$

xii. The field equations (70) with (68) are equivalent to ix., xi. and

$$
-h_{a b} R^{b c} h_{c d}=\left(\Lambda+\frac{1}{2} \rho\right) h_{a d} .
$$

(xi. arises through contraction of the field equations by $u^{a} u^{b}$, ix. with $u^{a} h^{b c}$, xii. with $h_{a c} h_{b d}$.)

From xi. there follows the noteworthy

Theorem 3.5.1. [44,45]. If along a flow line $\frac{1}{2} \Lambda+\omega^{2} \leq \frac{1}{4} \rho+\sigma^{2}$ (in particular thus for $\omega=0, \Lambda \leq 0$ ) and $\Theta(s)>0$ at a (proper-)time $s$, then there exists a (finite) $s_{0}<s$ such that $\lim _{s \rightarrow s_{0}+0}\left(\rho s^{2}\right)=+\infty$ : at the time $s=s_{0}$ a singular explosion has happened. For given $\Theta(s), s-s_{0}$, the 'age' of the geodesics, is increased by increasing $\omega$ or $\Lambda$, and is lowered by increasing $\sigma$ or $\rho$.

[These statements follow immediately from xi., if one observes that $\ddot{l}$ is the curvature of the graph of the function $l(s)$ and $\Theta=i / l$. For shearfree flows, $\sigma=0$, one can through ii. and viii. obtain from xi. an ordinary differential equation for $l(s)$. This equation can be integrated once.]

36 That is to say, vortex- and divergence-free; see Theorem 2.4.5.

37 This equation was derived by Raychaudhuri [44] in a special coordinate system. 
xiii. For shear-free flows there holds the simplified expansion equation

$$
3 l^{2}+\frac{2 \Omega^{2}}{l^{2}}-\frac{M}{l}-\Lambda l^{2} \equiv 10 E
$$

with parameters $\Omega, M$, and $E$ constant (along the flow lines).

In a vortex-free flow, (125) has the meaning of a condition for the curvature of the space-sections $t=$ const. $\left(u_{a} x^{a}=-d t\right)$. From the definitions of the quantities $\Theta, \sigma_{a b}$ it emerges that in this case $(\omega=0, \dot{u}=0)$ (up to a conventional factor) $\left(\sigma_{a b}+\frac{1}{3} \Theta h_{a b}\right) \delta_{\perp} x^{a} \delta_{\perp} x^{b}$ along $t=$ const. is the second fundamental form of these space sections, so that according to the formulae of differential geometry ${ }^{38}$ the left hand side of (124) can be reduced to the Ricci tensor of the space sections. Thus there holds

Theorem 3.5.2. In a vortex-free flow,

$$
K\left(x^{a}, e^{a}\right)=\frac{1}{3}\left(\Lambda+\rho+\sigma^{2}-\frac{1}{3} \Theta^{2}\right)+l^{-3}\left(l^{3} \sigma_{a b}\right) e^{a} e^{b}
$$

is the Gauss curvature of that surface $F$ which is generated by the flowlines of the geodesics (drawn with respect to the induced metric) going out from $x^{a}$, orthogonal to $e^{a}$ at $x^{a}$; where $e_{a} u^{a}=0$ (see Figure 2).

This theorem is a simple example of how in Einstein's theory the curvature of the rest-space of the matter is determined by its distribution and relative motion; the second term shows that form-altering motions of the matter inter alia are connected with an anisotropy of the spatial curvature (for the exceptions see Theorem 3.5.5.).

The equation (124) holds also in the Newtonian Mechanics of pressurefree matter interacting only through gravity; the relation (127) is the first typical of the Einstein theory. The first remark leads us to interpret the equation (126) as an energy theorem. In fact in Newtonian Theory the expansion energy, rotational energy, inner potential gravitational energy of a (small) matter sphere of radius $l$, consisting of substratum particles, are respectively given by the first three terms in (126) multiplied by $(2 \pi / 15) M$. If one adds to these a $\Lambda$-energy of magnitude $-(2 \pi / 15) M l^{2} \Lambda$, then (126) states the conservation of energy $(4 \pi / 3) l^{3} \rho E$ of this sphere. This mechanical energy theorem must however be distinguished from the thermodynamic energy theorem (78) (trivial for incoherent matter). The first concerns itself with a finitely extended system, and correspondingly contains a gravitational part, the second to an infinitesimal substratum

38 Equations of Gauss and Codacci, see e.g. Ref. 46 , sec.43 or Ref. 12, eq. (2.2.4a). 


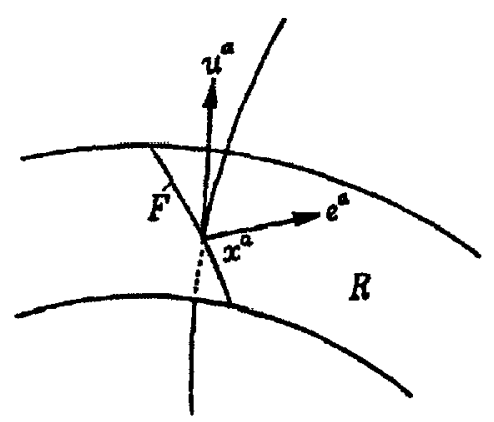

Figure 2.

particle, in which according to the equivalence principle gravitation does not make itself noticeable.

The dynamical conservation theorems viii. and xiii. holding for a matter sphere $K$ under the supposition $\sigma=0$, are valid although $K$ is not a closed system. On shrinking of $K$ through small spheres $k_{i}$ the energy does not behave linearly: the $E_{i}$ are related to local inertial systems moving relative to each other, and furthermore the $k_{i}$ interact through gravitation.

We now seek the kinematically simplest solutions of the gravitational equations for incoherent matter.

A. $(\omega=\Theta=\sigma=0)$ The simplest flow is a vortex- free rigid motion; this is by xi. and Theorem 3.5.2 only possible if $\rho=2 \Lambda$ and the restspace of the matter has the constant positive curvature $K=\Lambda$; and these conditions are by xii. and ix. also sufficient: Einstein-Universe.

B. $(\omega=\sigma=0, \Theta \neq 0)$ The next simplest possibility is that only $\sigma$ and $\omega$ vanish. Then (126) and (127) hold, and give the FriedmannLemaitre models. The rest spaces have the constant curvature $K=$ $-(10 / 3)\left(E / l^{2}\right) \equiv\left(k / R^{2}\right)(k= \pm 1,0)$. This curvature is thus equal to the above defined energy of a $l$-sphere, divided by $(4 / 10) \pi \rho l^{5}$. [That $\Theta$ and $\rho$ are constant in the rest spaces follows from (124) and (127)]. The theorems developed here and Theorem 2.5.1 permit, without further calculation, to draw the conclusion:

Theorem 3.5.3. (proved in Ref. 44 by other methods). The only universes with shear- and vortex-free motion of incoherent matter are those among the everywhere isotropic models characterised in Theorem 2.5.1, whose curvature radius obeys the Friedmann-Lemaitre equation (126) (with $l=R, \Omega=0,10 E=-3 k$ ). ${ }^{39}$

$3 \overline{9}$ For $\Lambda=0, k$ separates three classes of similar solutions (in the sense of Section 3.1) 
In anticipation of the application of these models to the system of extra-galactic nebulae, it is useful to replace the suppositions $\omega=0$ and $\sigma=0$ respectively by isotropy of the density distribution [48] $\left(h_{a}^{b} \rho_{, b}=0\right)$ and redshift [47]; from this namely follows according to 2.2.ii. and 2.2.vii. $\omega=0$ and $\sigma=0$. Both properties are in principle observable.

C. $(\Theta=\sigma=0, \omega \neq 0)$ Among the shear-free flows with rotation, the simplest are those that obey the conditions formulated in Theorem 2.5.2. For them there holds in suitable coordinates

$$
G=d z^{2}+d \sigma^{2}-\left(d t+u_{A}\left(x^{B}\right) d x^{A}\right)^{2}, \quad u^{a}=\delta_{4}^{a},
$$

where $d \sigma^{2}=\gamma_{A B}\left(x^{C}\right) d x^{A} d x^{B}$ is a surface metric. According to (125) necessarily $\frac{1}{2} \rho=\Lambda+2 \omega^{2}$. Therefore according to Theorem 2.5.2 $\rho=$ const. and $\omega=$ const. must hold. Since $G$ is the direct sum (see e.g. Ref. 12, Ch.1.4.) of the $z$-line and a $V_{3}$, from (125) necessarily $\Lambda+\frac{1}{2}=0$ must hold. Thus there follows $\rho=2 \omega^{2}=-2 \Lambda$, and to show that $\omega_{a}$ is constant one need only further solve the equation $h_{a b} R^{b c} h_{c d}=0$ for (64), which for example can be easily done with the help of the formulae in Ref. 12 (eq. (2.3.3), p.36), and then gives the Gödel cosmos [17,47,49-51].

Theorem 3.5.4. The Gödel cosmos is the only universe with incoherent matter in which the motion of the substratum proceeds shear-free, and the direction of the rotation axis in the infinitesimal spacelike neighbourhood of each particle is constant.

As the theorem shows, a rigid, spatially-constant rotation is only consistent with the field equations for $\Lambda<0$; the rotation must therefore only be so strong, that the matter can be held together by the (usual) gravitational and $\Lambda$-attraction. If one however does not require constancy of $\omega$, there are permitted for $\Lambda=0$ flow fields with rigid rotations that with the help of remarks v.,viii.,ix.,xi.,xii. can be reduced to the static solutions of the vacuum field equations $R_{a b}=0$ in a surprisingly simple way (Ref. 47 , $\S 29$, Theorem IV). Since this has already been presented completely in the spirit of the methods used here (Ref. 52; see also Ref. 53), we refrain from a repetition and simply remark: In these solutions, $\rho=4 \omega^{2} ; \rho>0$ therefore does not have to be assumed separately. Solutions with (spatially) constant density do not exist (Ref. $47, \S 29$, Theorem III)-contrary to the Newtonian theory, where from $\Theta=\sigma=0$ it follows that $\rho=$ const. (Ref. 41, I), just as there exist no solutions with compact orientable 3space (Ref. $47, \S 29$, Theorem IV). Axially symmetric solutions of this kind have been constructed-without reference to vacuum fields-by van

from each other; in each class, $M$ is a similarity parameter. 
Stockum [54]. This author also found the only singularity-free solution of this kind known up to now, a rotating cylinder with a static (!) vacuum field attached to it; solutions that contain singular points, surfaces, and so on can be obtained in great numbers (Ref. $47, \S 29$, Theorem IV). It is an interesting question, whether these singularities can be eliminated through introduction of physically acceptable pressures, or through joining to a vacuum field.

D. $(\omega=0)$. The next simplest flow is one that is vortex-free and volume-preserving; according to xi. this is at best possible for $\Lambda=\frac{1}{2} \rho+2 \sigma^{2}$ $(>0)$. Solutions of this type [except for $\sigma=0$, see (A)] have not been given, to my knowledge. Under the more complicated assumptions $\omega=$ $0, \Theta \neq 0 \neq \sigma$, firstly the spherically symmetric flows can be specified, and have been exhaustively investigated by several authors $[55,56]$; in the framework of this presentation, they can be lucidly handled with the help of statements xi, ix. and 3.5.2.

From Theorem 3.5.2, ix. and xi. follows the

Theorem 3.5.5. In a vortex-free flow of incoherent matter, the rest-spaces of the substratum are of constant curvature $K(t)$ if and only if the tensor $l^{3} \sigma_{a b}$ is constant along the flow lines. For such flows the Einstein equations are equivalent to ix., xi. (for $\omega=0$ ) and

$$
K(t)=\frac{k}{R^{2}}=\frac{1}{3}\left(\Lambda+\rho+\sigma^{2}-\frac{1}{3} \Theta^{2}\right) .
$$

The corresponding flows are natural generalisations of the FriedmannLemaitre models: examples of this kind have been determined (from another viewpoint and using other methods) by Schücking and Heckmann, see Ref. 9.

It is clear that in the characterisation of more general flows, the vanishing or not of kinematic quantities or their constancy is not sufficient, rather further invariants must be introduced; and that for $\omega \neq 0$ the metric determined by $h_{a b}$ should be considered as the metric of the three-dimensional space of the flow-lines, for which circumstances theorems analogous to 3.5.2 should be formulated. We will not investigate these questions here; our aim was to show the simplicity and applicability of geometrically formulated hydrodynamics in Riemannian space for the handling of exact solutions, and presentation of the simplest examples. ${ }^{40}$

40 More general solutions, that are spatially homogeneous, have been determined by Heckmann, Schücking, and Ozsvath; these works have not yet been published (private communication). 


\section{REFERENCES}

1. Landau, L. D., and Lifschitz, E. M. (1962) The Classical Theory of Fields (AddisonWesley, Reading, Mass.) [also reprinted (1975). (Pergamon Press, Oxford)].

2. Lichnerowicz, A. (1955). Théories relativistes de la gravitation et de l'électromagnétisme (Masson, Paris)..

3. Synge, J. L. (1937). Proc. Lond. Math. Soc. 43.

4. Salzman, G., and Taub, A. H. (1954). Phys. Rev. 95, 1659.

5. Jordan, P., Ehlers, J., Sachs, R. K. (1961). Akad. Wiss. Mainz. Abh. math.-nat. Kl. 1961 Nr. 1.

6. Synge, J. L., and Schild, A. (1952). Tensor Calculus (University of Toronto Press, Toronto) [also reprinted (1978). (Dover, New York)].

7. Foures-Bruhat, Y. (1958). Comptes Rend. Acad. Sci. (Paris) II 246, 3319.

8. Gödel, K. (1952). In Proc. Int. Congress of Mathematicians (Cambridge, Mass., Aug./Sept. 1950), (Int. Congress Mathematicians/A.M.S., Providence, R.I.) vol. 1.

9. Schücking, E., and Heckmann, O. (1959). In Onzième Conseil de Physique Solvay: La Structure et l'Évolution de l'Univers, Int. Inst. de Physique Solvay (Editions Stoop, Brussels).

10. Rayner, C. B. (1959). Comptes Rend. Acad. Sci. (Paris) II 248, 929, 2725; 249, 1327.

11. Pirani, F. A. E. (1957). Phys. Rev. 105, 1089.

12. Jordan, P., Ehlers, J., and Kundt, W. (1960). Akad. Wiss. Mainz. Abh. math.-nat. Kl. $1961 \mathrm{Nr} .2$.

13. Just, K. (1956). Z. Physik. 145, 235.

14. Robertson, H. P. (1933). Rev. Mod. Phys. 5, 62.

15. Bondi, H. (1960). Cosmology (Cambridge University Press, Cambridge).

16. Einstein, A. (1949). The Meaning of Relativity (3rd. ed., Princeton University Press, Princeton).

17. Gödel, K. (1949). Rev. Mod. Phys. 21, 447.

18. Weyl, H. (1921/1923/1970/1988). Raum Zeit Materie (4th ed., 5th ed., 6th ed., 7th ed., Springer-Verlag, Berlin) [English transl. reprinted (1952). Space-Time-Matter (Dover, New York)].

19. Pauli, W. (1921). "Relativitätstheorie" In Enzykl. d. Math. Wiss. (Teubner, Leipzig) vol. 5, part 2, p. 539 [English transl. with supplements (1958). Theory of Relativity (Pergamon Press, London/Oxford)].

20. Eckart, C. (1940). Phys. Rev. 58, 919.

21. Meixner, J., and Reik, W. (1959). In Handbuch der Physik III, 2 (Springer-Verlag, Berlin).

22. Just, K. (1958). Habilitation Thesis, FU Berlin.

23. Birkhoff, G. (1950). Hydrodynamics (Princeton University Press, Princeton).

24. Eisenhart, L. P. (1924). Trans. Amer. Math. Soc. 26, 205.

25. Jordan, P. (1955). Schwerkraft und Weltall (2nd. ed., Vieweg \& Sohn, Braunschweig).

26. Fierz, M. (1956). Helv. Phys. Act. 29, 128.

27. Heckmann, O., and Schücking, E. (1959). In Handbuch der Physik LIII, 519 (??, Berlin).

28. Pirani, F. A. E. (1956). Acta Phys. Polonica 15, 389.

29. Fock, V. (1960). Theorie von Raum, Zeit und Gravitation (Akademie-Verlag, Berlin) [English transl. by N. N. Kemmer (1964). The Theory of Space, Time and Gravitation (2nd. rev. ed., Macmillan, New York)]. 
30. Whittaker, E. T. (1935). Proc. Roy. Soc. Lond. 149, 384.

31. Bondi, H., Pirani, F. A. E., and Robinson, I. (1959). Proc. Roy. Soc. Lond. A251, 519.

32. Witten L., ed. (1962). The Theory of Gravitation: An Introduction to Current Research (Wiley, New York).

33. Sasaki, M. (1958). In Max Planck Festschrift (VEB Deutscher Verlag der Wissenschaften, Berlin).

34. Grad, H. (1949). Comm. Pure Appl. Math. 2, 331.

35. Synge, J. L. (1960). Relativity: The General Theory (North-Holland, Amsterdam).

36. Grad, H. (1959). In Handbuch der Physik XII (Springer-Verlag, Berlin).

37. Taub, A. H. (1948). Phys. Rev. 74, 328.

38. Taub, A. H. (1956). Phys. Rev. 103, 454.

39. Boltzmann, L. (1895). Vorlesungen über Gastheorie (Barth, Leipzig) [English transl. by S. G. Brush (1964). Lectures on Gas Theory (University of California Press, Berkeley)].

40. Heckmann, O. (1942). Theorien der Kosmologie (Springer-Verlag, Berlin).

41. Heckmann, O., and Schücking, E. (1955). Z. Ap. 38, 95 (I); (1956). 40, 81 (II).

42. Tolman, R. C. (1934). Relativity, Thermodynamics and Cosmology (Oxford University Press, Oxford) [also reprinted (1987). (Dover, New York)].

43. Pound, R. V., and Rebka, G. A. (1960). Phys. Rev. Lett. 4, 337.

44. Raychaudhuri, A. (1955). Phys. Rev. 98, 1123.

45. Komar, A. (1956). Phys. Rev. 104, 544.

46. Eisenhart, L. P. (1956). Riemannian Geometry Princeton University Press, Princeton).

47. Ehlers, J. (1957). Dissertation, Hamburg University.

48. Bondi, H. (1947). Mon. Not. R. Astr. Soc. 107, 410.

49. Kundt, W. (1955). Diploma Thesis, Hamburg University.

50. Kundt, W. (1956). Z. Phys. 145, 611.

51. Einstein, A. (ed. P. A. Schilpp) (1949). Albert Einstein: Philosopher-Scientist (Library of Living Philosophers, Tudor, New York).

52. Ehlers, J. (1959). In Les Théories relativistes de la gravitation (Actes du Colloque Int., Royaumont, 21-27 Juin 1959) (CNRS, Paris).

53. Ehlers, J. (1962). In Recent Developments in General Relativity (Dedicated to Infeld) (Pergamon Press, Oxford, and PWN-Polish Scientific Publishers, Warsaw).

54. van Stockum, W. J. (1937). Proc. R. Soc. Edinburgh 57, 135. 